\title{
Reduced measures for semilinear elliptic equations involving Dirichlet operators
}

\author{
Tomasz Klimsiak ${ }^{1,2}$
}

Received: 5 November 2015 / Accepted: 28 May 2016/ Published online: 21 June 2016

(C) The Author(s) 2016. This article is published with open access at Springerlink.com

\begin{abstract}
We consider elliptic equations of the form (E) $-A u=f(x, u)+\mu$, where $A$ is a negative definite self-adjoint Dirichlet operator, $f$ is a function which is continuous and nonincreasing with respect to $u$ and $\mu$ is a Borel measure of finite potential. We introduce a probabilistic definition of a solution of (E), develop the theory of good and reduced measures introduced by H. Brezis, M. Marcus and A.C. Ponce in the case where $A=\Delta$ and show basic properties of solutions of (E). We also prove Kato's type inequality. Finally, we characterize the set of good measures in case $f(u)=-u^{p}$ for some $p>1$.
\end{abstract}

Mathematics Subject Classification $35 \mathrm{~J} 75 \cdot 60 \mathrm{~J} 45$

\section{Introduction}

Let $E$ be a separable locally compact metric space and let $m$ be a Radon measure on $E$ such that $\operatorname{supp}[m]=E$. In the present paper we study semilinear equations of the form

$$
-A u=f(x, u)+\mu,
$$

where $\mu$ is a Borel measure on $E, f: E \times \mathbb{R} \rightarrow \mathbb{R}$ is a measurable function such that $f(\cdot, u)=0, u \leq 0$, and $f$ is nonincreasing and continuous with respect to $u$. As for the operator $A$, we assume that it is a negative definite self-adjoint Dirichlet operator on $L^{2}(E ; m)$. Saying that $A$ is a Dirichlet operator we mean that

$$
\left(A u,(u-1)^{+}\right) \leq 0, \quad u \in D(A) .
$$

Communicated by H. Brezis.

Tomasz Klimsiak tomas@mat.umk.pl

1 Institute of Mathematics, Polish Academy of Sciences, Śniadeckich 8, 00-956 Warszawa, Poland

2 Faculty of Mathematics and Computer Science, Nicolaus Copernicus University, Chopina 12/18, 87-100 Toruń, Poland 
Equivalently, operator $A$ corresponds to some symmetric Dirichlet form $(\mathcal{E}, D[\mathcal{E}])$ on $L^{2}(E ; m)$ in the sense that

$$
D(A) \subset D[\mathcal{E}], \quad \mathcal{E}(u, v)=(-A u, v), \quad u \in D(A), v \in D[\mathcal{E}]
$$

(see $[12,23])$ The class of such operators is quite large. It contains many local as well as nonlocal operators. The model examples are Laplace operator $\Delta$ (or uniformly elliptic divergence form operator) and the fractional Laplacian $\Delta^{\alpha}$ with $\alpha \in(0,1)$. Many other examples are to be found in $[12,23]$.

Let Cap denote the capacity determined by $(\mathcal{E}, D[\mathcal{E}])$ (see Sect. 2). It is known (see [13]) that any Borel signed measure $\mu$ on $E$ admits a decomposition

$$
\mu=\mu_{c}+\mu_{d}
$$

into the singular (concentrated) part $\mu_{c}$ with respect to Cap and the absolutely continuous (diffuse, smooth) part $\mu_{d}$ with respect to Cap. The smooth part $\mu_{d}$ is fully characterized in [20].

The study of semilinear equations of the form (1.1) in case $\mu$ is smooth, i.e. when $\mu_{c}=0$, goes back to the papers by Brezis and Strauss [7] and Konishi [21] In [7,21] the existence of a solution of (1.1) is proved for $\mu \in L^{1}(E ; m)$. At present existence, uniqueness and regularity results are available for equation (1.1) involving general bounded smooth measure $\mu$ and operator corresponding to Dirichlet form (see Klimsiak and Rozkosz [17] for the case of symmetric regular Dirichlet form and [19] for the case of quasi-regular, possibly non-symmetric Dirichlet form). The case $\mu_{c} \neq 0$ is much more involved. Ph. Bénilan and $\mathrm{H}$. Brezis [2] has observed that in such a case equation (1.1) need not have a solution even if $A=\Delta$. In [5] (see also [4]) H. Brezis, M. Marcus and A.C. Ponce introduced the concept of good measure, i.e. a bounded measure for which (1.1) has a solution, and the concept of reduced measure, i.e. the largest good measure, which is less then or equal to $\mu$. In case $A=\Delta$ these concepts are by now quite well investigated (see [2,5]). The situation is entirely different in case of more general local operators or nonlocal operators. There are known, however, some existence and uniqueness results for (1.1) in case $A$ is a diffusion operator (see Véron [28]) and in case $A=\Delta^{\alpha}$ with $\alpha \in(0,1)$ (see Chen and Véron [8]).

The main purpose of the paper is to present a new approach to (1.1) that provides a unified way of treating (1.1) for the whole class of negative defined self-adjoint Dirichlet operators $A$ and for $\mu$ from some class of measures $\mathbb{M}$ including the class $\mathcal{M}_{b}$ of bounded signed Borel measures on $E$. In particular, we give a new definition of a solution of (1.1) and investigate the structure of good and reduced measures relative to (1.1). In case $A=\Delta$ our definition is equivalent to the definition of a solution adopted in $[2,5]$, so our results generalize the results of $[2,5]$ to wide class of operators. In fact, they generalize the existing results even in case $A=\Delta$, because in this case $\mathcal{M}_{b} \varsubsetneqq \mathbb{M}$ and $\mathbb{M}$ contains important in applications unbounded measures. The second purpose of our paper is to give a probabilistic interpretation for solutions of (1.1).

First, some remarks concerning our definition of a solution and the class $\mathbb{M}$ are in order. Suppose we want to consider problem (1.1) for some class of measures $\mathbb{M}$ including $L^{1}(E ; m)$. Considering $f \equiv 0$ in (1.1) we see that then $G:=-A^{-1}$ should be well defined on $L^{1}(E ; m)$, i.e. the following condition should be satisfied:

$$
G g \equiv \uparrow \lim _{N \rightarrow \infty} \int_{0}^{N} T_{t} g d t<\infty, \quad m \text {-a.e., } \quad g \in L^{1,+}(E ; m) .
$$


Condition (1.3) is nothing but the statement that the semigroup $\left\{T_{t}, t \geq 0\right\}$ generated by $A$ (or, equivalently, the Dirichlet form $(\mathcal{E}, D[\mathcal{E}])$ ) is transient (see [12, Section 1.5]). It is well known that then there exists a kernel $\{R(x, d y), x \in E\}$ such that for every $g \in L^{1,+}(E ; m)$,

$$
\int_{E} g(y) R(\cdot, d y)=G g, \quad m \text {-a.e. }
$$

If $u$ is a solution of (1.1) with $f \equiv 0$ then

$$
u \cdot m(d x)=R \circ \mu(d x)
$$

where $R \circ \mu$ is a Borel measure defined as

$$
\int_{E} g(x)(R \circ \mu)(d x)=\int_{E} \int_{E} g(y) R(x, d y) \mu(d x), \quad g \in \mathcal{B}^{+}(E) .
$$

Therefore $R \circ \mu$ must be absolutely continuous with respect to the measure $m$ for every bounded Borel measure $\mu$. This condition is known in the literature as the Meyer hypothesis (L) (see [3]) or the condition of absolute continuity of the resolvent $\left\{G_{\alpha}, \alpha>0\right\}$ (see [12]).

For the reasons explained above in the paper we assume that $\left\{T_{t}, t \geq 0\right\}$ is transient and hypothesis (L) is satisfied. It is known that under these assumptions there exists a Borel function $r: E \times E \rightarrow \mathbb{R}_{+}$such that

$$
r(x, y) m(d y)=R(x, d y), \quad x \in E .
$$

Using the kernel $r$ we can give our first, purely analytical definition of a solution of (1.1). Namely, we say that a Borel function $u$ on $E$ is a solution of (1.1) if

$$
u(x)=\int_{E} f(y, u(y)) r(x, y) d y+\int_{E} r(x, y) \mu(d y)
$$

for $m$-a.e. $x \in E$. Of course, to make this definition correct we have to assume that the integrals in (1.4) exist. Therefore the class $\mathbb{M}$ we consider consists of Borel measures $\mu$ on $E$ such that $\int_{E} r(x, y)|\mu|(d y)<\infty$ for $m$-a.e. $x \in E$. We will show that $\mathcal{M}_{b}(E) \subset \mathbb{M}$. In general, the inclusion is strict. For instance, if $A=\Delta^{\alpha}, \alpha \in(0,1]$, on an open set $D \subset \mathbb{R}^{d}$, then $\mathbb{M}$ includes the set of all Borel measures $\mu$ on $E$ such that $\delta^{\alpha} \cdot \mu \in \mathcal{M}_{b}$, where $\delta(x)=\operatorname{dist}(x, \partial D)$. We also show that in case $\mu \in \mathcal{M}_{b}$ and $A$ is a uniformly elliptic divergence form operator on a bounded domain in $\mathbb{R}^{d}$ definition (1.4) is equivalent to Stampacchia's definition by duality (see [27]).

Unfortunately, definition (1.4) is rather inconvenient for studying (1.1). One of the main results of the paper says that (1.4) is equivalent to our second, probabilistic in nature definition of a solution. At first glance the probabilistic definition seems to be more complicated than (1.4), but as a matter of fact suits much better to the purposes of the present paper. Let $\mathbb{X}=\left(\left\{X_{t}, t \geq 0\right\},\left\{P_{x}, x \in E\right\}\right)$ be a Hunt process with life time $\zeta$ associated with the form $(\mathcal{E}, D[\mathcal{E}])$. We say that $u$ is a probabilistic solution of (1.1) if

(a) $f(\cdot, u) \cdot m \in \mathbb{M}$ and there exists a local martingale additive functional $M$ of $\mathbb{X}$ such that

$$
u\left(X_{t}\right)=u\left(X_{0}\right)-\int_{0}^{t} f\left(X_{r}, u\left(X_{r}\right)\right) d r-\int_{0}^{t} d A_{r}^{\mu_{d}}+\int_{0}^{t} d M_{r}, \quad t \geq 0, \quad P_{x} \text {-a.s. }
$$

for quasi every (q.e. for short) $x \in E$ (Here $A^{\mu_{d}}$ denotes a continuous additive functional of $\mathbb{X}$ of finite variation in the Revuz correspondence with $\mu_{d}$ ), 
(b) for every polar set $N \subset E$, every stopping time $T \geq \zeta$ and every sequence of stopping times $\left\{\tau_{k}\right\}$ such that $\tau_{k} \nearrow T$ and $E_{x} \sup _{t \leq \tau_{k}}\left|u\left(X_{t}\right)\right|<\infty$ for $x \in E \backslash N$ and $k \geq 1$ we have

$$
E_{x} u\left(X_{\tau_{k}}\right) \rightarrow R \mu_{c}(x), \quad x \in E \backslash N,
$$

where $E_{x}$ denotes the integration with respect to probability $P_{x}$ and

$$
R \mu_{c}(x)=\int_{E} r(x, y) \mu_{c}(d y), \quad x \in E .
$$

The above probabilistic definition allows us to develop a general theory of equations of the form (1.1). Moreover, in our opinion, the theory based on the probabilistic definition is elegant and simple.

We first prove some regularity results. We show that if $u$ is a solution of (1.1) and $\mu \in \mathcal{M}_{b}$ then $T_{k}(u) \in D_{e}[\mathcal{E}]$ and

$$
\mathcal{E}\left(T_{k}(u), T_{k}(u)\right) \leq 2 k\|\mu\|_{T V}, \quad k \geq 0,
$$

where $T_{k}(u)=\min \{\max \{u,-k\}, k\}$ and $D_{e}[\mathcal{E}]$ is an extension of the domain of the form $\mathcal{E}$ such that the pair $\left(\mathcal{E}, D_{e}[\mathcal{E}]\right)$ is a Hilbert space (see [12]). We also prove Stampacchia's type inequality which says that for every strictly positive excessive function $\rho$ (for $\rho \equiv 1$ for instance) and $\mu \in \mathcal{M}_{\rho}=\left\{\mu \in \mathbb{M}:\|\mu\|_{T V, \rho}:=\|\rho \cdot \mu\|_{T V}<\infty\right\}$,

$$
\|f(\cdot, u)\|_{L^{1}(E ; \rho \cdot m)} \leq\|\mu\|_{T V, \rho} .
$$

We next study the structure of the set $\mathcal{G}$ of good measures and the set of reduced measures relative to $A, f$. Let us recall that the reduced measure is the largest measure $\mu^{*} \in \mathbb{M}$ such that $\mu^{*} \leq \mu$ and there exists a solution of (1.1) with $\mu$ replaced by $\mu^{*}$. A measure $\mu \in \mathbb{M}$ is good, if $\mu^{*}=\mu$. By results of $[17,19]$, if $\mu_{c}=0$, then $\mu$ is good. In the present paper we first show that

$$
\mu-\mu^{*} \perp \text { Cap. }
$$

Then we show that, as in the case of Laplace operator, the set $\mathcal{G}$ is convex and closed under the operation of taking maximum of two measures. We also show that $\mu \in \mathcal{G}$ if and only if

$$
\mu=g-A v
$$

for some functions $g, v$ on $E$ such that $g \cdot m, f(\cdot, v) \cdot m \in \mathbb{M}$ and $A v \in \mathbb{M}$. From this characterization of $\mathcal{G}$ we deduce that for every strictly positive excessive function $\rho$,

$$
L^{1}(E ; \rho \cdot m)+\mathcal{A}_{\rho}(f)=\mathcal{G} \cap \mathcal{M}_{\rho},
$$

where

$$
\mathcal{A}_{\rho}(f)=\left\{\mu \in \mathcal{M}_{\rho}: f(\cdot, R \mu) \in L^{1}(E ; \rho \cdot m)\right\} .
$$

We also show that under some additional assumption on the growth of $f$ (it is satisfied for instance if $|f(x, u)| \leq c_{1}+c_{2} e^{u^{2}}$, for every strictly positive excessive function $\rho$,

$$
\overline{\mathcal{A}_{\rho}(f)}=\mathcal{G} \cap \mathcal{M}_{\rho},
$$

where the closure is taken in the space $\left(\mathcal{M}_{\rho},\|\cdot\|_{T V, \rho}\right)$.

In Sect. 6 we prove the so-called inverse maximum principle and Kato's type inequality. In our context Kato's inequality says that if $u$ is a solution of (1.1) then $A u^{+} \in \mathbb{M}$ and

$$
\mathbf{1}_{\{u>0\}}(A u)_{d} \leq\left(A u^{+}\right)_{d}, \quad(A u)_{c}^{+}=\left(A u^{+}\right)_{c} .
$$


This form of Kato's inequality for Laplace operator was proved by H. Brezis and A.C. Ponce in [6].

In the last section we study the set of good measures $\mathcal{G}$ for problem (1.1) with $f$ having at most polynomial growth, i.e. for $f$ satisfying

$$
|f(x, u)| \leq c|u|^{p}, \quad x \in E, u \geq 0
$$

for some $p>1$. For this purpose, we introduce a new capacity $\operatorname{Cap}_{A, p}$, which in the special case, when $A=\Delta^{\alpha}$ on an open bounded set $D \subset \mathbb{R}^{d}$ with zero boundary condition is equivalent to the Bessel capacity defined as

$$
\operatorname{Cap}_{\alpha, p}^{D}(K)=\inf \left\{\|\eta\|_{W^{2 \alpha, p}(D)}^{p}: \eta \in C_{c}^{\infty}(D), \eta \geq \mathbf{1}_{K}\right\}
$$

for compact sets $K \subset D$. We prove that if $\mu \in \mathbb{M}$ and $\mu^{+}$is absolutely continuous with respect to $\operatorname{Cap}_{A, p^{\prime}}$, where $p^{\prime}$ denotes the Hölder conjugate to $p$, then a solution of (1.1) exists, i.e. $\mu \in \mathcal{G}$. For $f$ of the form

$$
f(x, u)=-u^{p}, \quad x \in E, u \geq 0
$$

we fully characterize the set $\mathcal{G}$. Namely, we prove that the absolute continuity of $\mu^{+}$with respect to $\mathrm{Cap}_{A, p^{\prime}}$ is also necessary for the existence of a solution of (1.1). Thus, in case $f$ is given by (1.6),

$$
\mathcal{G}=\left\{\mu \in \mathbb{M}: \mu^{+} \ll \operatorname{Cap}_{\alpha, p^{\prime}}^{D}\right\}
$$

Moreover,

$$
\mu^{*}=\mu_{\mathrm{Cap}_{A, p^{\prime}}^{+}}^{+}-\mu^{-},
$$

where $\mu_{\mathrm{Cap}_{A, p^{\prime}}}^{+}$denotes the absolutely continuous part of $\mu^{+}$with respect to $\mathrm{Cap}_{A, p^{\prime}}$.

\section{Preliminaries}

In the paper $E$ is a locally compact separable metric space and $m$ is a positive Radon measure on $E$ such that $\operatorname{supp}[m]=E$. By $(\mathcal{E}, D[\mathcal{E}])$ we denote a symmetric regular Dirichlet form on $L^{2}(E ; m)$ (see [12] or [23] for the definitions). We will always assume that $(\mathcal{E}, D[\mathcal{E}])$ is transient, i.e. there exists a strictly positive function $g$ on $E$ such that

$$
\int_{E}|u(x)| g(x) m(d x) \leq\|u\|_{\mathcal{E}}, \quad u \in D[\mathcal{E}]
$$

where $\|u\|_{\mathcal{E}}=\sqrt{\mathcal{E}(u, u)}, u \in D[\mathcal{E}]$. As usual, for $\alpha>0$ we set $\mathcal{E}_{\alpha}(u, v)=\mathcal{E}(u, v)+\alpha(u, v)$, $u, v \in D[\mathcal{E}]$, where $(\cdot, \cdot)$ is the usual inner product in $L^{2}(E ; m)$.

By Riesz's theorem, for every $\alpha>0$ and $f \in L^{2}(E ; m)$ there exists a unique function $G_{\alpha} f \in L^{2}(E ; m)$ such that

$$
\mathcal{E}_{\alpha}\left(G_{\alpha} f, g\right)=(f, g), \quad g \in L^{2}(E ; m) .
$$

It is an elementary check that $\left\{G_{\alpha}, \alpha>0\right\}$ is a strongly continuous contraction resolvent on $L^{2}(E ; m)$. By $\left\{T_{t}, t \geq 0\right\}$ we denote the associated semigroup and by $(A, D(A))$ the self-adjoint negative definite Dirichlet operator generated by $\left\{T_{t}\right\}$. It is well known that $A$ satisfies (1.2) (see [12, Section 1.3]). Conversely, one can prove (see [23, page 39]) that for 
every self-adjoint negative definite Dirichlet operator $A$ there exists a unique Dirichlet form $(\mathcal{E}, D[\mathcal{E}])$ such that $(1.2)$ holds.

Given a Dirichlet form $(\mathcal{E}, D[\mathcal{E}])$ we define capacity Cap: $2^{E} \rightarrow \mathbb{R}^{+}$as follows: for an open $U \subset E$ we set

$$
\operatorname{Cap}(U)=\inf \left\{\mathcal{E}(u, u): u \in D[\mathcal{E}], u \geq \mathbf{1}_{U}, m \text {-a.e. }\right\}
$$

and then for arbitrary $A \subset E$ we set

$$
\operatorname{Cap}(A)=\inf \{\operatorname{Cap}(U): A \subset U \subset E, U \text { open }\} .
$$

An increasing sequence $\left\{F_{n}\right\}$ of closed subsets of $E$ is called nest if $\operatorname{Cap}\left(E \backslash F_{n}\right) \rightarrow 0$ as $n \rightarrow \infty$. A subset $N \subset E$ is called exceptional if $\operatorname{Cap}(N)=0$. We say that some property $P$ holds quasi everywhere (q.e. for short) if a set for which it does not hold is exceptional.

We say that a function $u$ on $E$ is quasi-continuous if there exists a nest $\left\{F_{n}\right\}$ such that $u_{\mid F_{n}}$ is continuous for every $n \geq 1$. It is known that each function $u \in D[\mathcal{E}]$ has a quasi-continuous $m$-version.

A Borel measure $\mu$ on $E$ is called smooth if it does not charge exceptional sets and there exists a nest $\left\{F_{n}\right\}$ such that $|\mu|\left(F_{n}\right)<\infty, n \geq 1$. By $S$ we denote the set of all smooth measures on $E$.

By $S_{0}^{(0)}$ we denote the set of all measures $\mu \in S$ for which there exists $c>0$ such that

$$
\int_{E}|u| d|\mu| \leq c \sqrt{\mathcal{E}(u, u)}, \quad u \in D[\mathcal{E}] .
$$

For a given Dirichlet form $(\mathcal{E}, D[\mathcal{E}])$ one can always define the so-called extended Dirichlet space $D_{e}[\mathcal{E}]$ as the set of $m$-measurable functions on $E$ for which there exists an $\mathcal{E}$-Cauchy sequence $\left\{u_{n}\right\} \subset D[\mathcal{E}]$ convergent $m$-a.e. to $u$ (the so-called approximating sequence). One can show that for $u \in D_{e}[\mathcal{E}]$ the limit $\mathcal{E}(u, u)=\lim _{n \rightarrow \infty} \mathcal{E}\left(u_{n}, u_{n}\right)$ exists and does not depend on the approximating sequence $\left\{u_{n}\right\}$ for $u$. Each element $u \in D_{e}[\mathcal{E}]$ has a quasicontinuous version. It is known that $(\mathcal{E}, D[\mathcal{E}])$ is transient if and only if $\left(\mathcal{E}, D_{e}[\mathcal{E}]\right)$ is a Hilbert space. In the latter case for a given measure $\mu \in S_{0}^{(0)}$ inequality (2.1) holds for every $u \in D_{e}[\mathcal{E}]$.

By $\mathcal{M}_{b}$ we denote the set of all bounded Borel measures on $E$ and by $\mathcal{M}_{0, b}$ the subset of $\mathcal{M}_{b}$ consisting of smooth measures.

Given a Borel measurable function $\eta$ on $E$ and a Borel measure $\mu$ on $E$ we write

$$
(\mu, \eta)=\int_{E} \eta d \mu
$$

By $u \cdot \mu$ w denote the Borel measure on $E$ defined as

$$
(f, u \cdot \mu)=(f \cdot u, \mu), \quad f \in \mathcal{B}(E)
$$

whenever the integrals exist.

With a regular symmetric Dirichlet form $(\mathcal{E}, D[\mathcal{E}])$ one can associate uniquely a symmetric Hunt process $\mathbb{X}=\left(\left(X_{t}\right)_{t \geq 0},\left(P_{x}\right)_{x \in E},\left(\mathcal{F}_{t}\right)_{t \geq 0}, \zeta\right)$ (see [12, Section 7.2]). It is related to $(\mathcal{E}, D[\mathcal{E}])$ by the formula

$$
T_{t} f(x)=E_{x} f\left(X_{t}\right), \quad t \geq 0, \quad m \text {-a.e. }
$$

where $E_{x}$ stands for the expectation with respect to the measure $P_{x}$. For $\alpha, t \geq 0$ and $f \in \mathcal{B}^{+}(E)$ we write

$$
R_{\alpha} f(x)=E_{x} \int_{0}^{\zeta} e^{-\alpha t} f\left(X_{t}\right) d t, \quad p_{t} f(x)=E_{x} f\left(X_{t}\right), \quad x \in E .
$$


Observe that for $\alpha, t>0$ and $f \in L^{2}(E ; m)$,

$$
R_{\alpha} f=G_{\alpha} f, \quad p_{t} f=T_{t} f, \quad m \text {-a.e. }
$$

For simplicity we denote $R_{0}$ by $R$. We say that some function on $E$ is measurable if it is universally measurable, i.e. measurable with respect to the $\sigma$-algebra

$$
\mathcal{B}^{*}(E)=\bigcap_{\mu \in \mathcal{P}(E)} \mathcal{B}^{\mu}(E),
$$

where $\mathcal{P}(E)$ is the set of all probability measures on $E$ and $\mathcal{B}^{\mu}(E)$ is the completion of $\mathcal{B}(E)$ with respect to the measure $\mu$.

A positive measurable function $u$ on $E$ is called $\alpha$-excessive if for every $\beta>0,(\alpha+$ $\beta) R_{\alpha+\beta} u \leq u$ and $\alpha R_{\alpha} u \nearrow u$ as $\alpha \rightarrow \infty$. By $\mathcal{S}_{\alpha}$ we denote the set of $\alpha$-excessive functions. We put $\mathcal{S}=\mathcal{S}_{0}$.

By $S_{00}^{(0)}$ we denote the set of all $\mu \in S_{0}^{(0)}$ such that $|\mu|(E)<\infty$ and $R|\mu|$ is bounded. For a Borel set $B$ we set

$$
\sigma_{B}=\inf \left\{t>0 ; X_{t} \in B\right\}, \quad D_{A}=\inf \left\{t \geq 0 ; X_{t} \in B\right\}, \quad \tau_{B}=\sigma_{E \backslash B},
$$

i.e. $\sigma_{B}$ is the first hitting time of $B, D_{A}$ is the first debut time of $B$ and $\tau_{B}$ is the first exit time of $B$.

By $\mathcal{T}$ we denote the set of all stopping times with respect to the filtration $\left(\mathcal{F}_{t}\right)_{t \geq 0}$ and by D the set of all measurable functions $u$ on $E$ for which the family

$$
\left\{u\left(X_{\tau}\right), \tau \in \mathcal{T}\right\}
$$

is uniformly integrable with respect to the measure $P_{x}$ for q.e. $x \in E$.

For a Borel measure $\mu$ on $E$ and $\alpha \geq 0$ we denote by $\mu \circ R_{\alpha}$ the measure defined as

$$
\left(f, \mu \circ R_{\alpha}\right)=\left(R_{\alpha} f, \mu\right), \quad f \in \mathcal{B}(E),
$$

and by $P_{\mu}$ we denote the measure

$$
P_{\mu}(A)=\int_{E} P_{x}(A) \mu(d x), \quad A \in \mathcal{F}_{\infty} .
$$

In the whole paper we assume that $m$ is the reference measure for $\mathbb{X}$, i.e. for all $x \in E$ and $\alpha>0$ we have $R_{\alpha}(x, \cdot) \ll m$. It is well known (see [12, Lemma 4.2.4]) that in this case for every $\alpha \geq 0$ there exists a $\mathcal{B}(E) \otimes \mathcal{B}(E)$ measurable function

$$
r_{\alpha}: E \times E \rightarrow \mathbb{R}^{+}
$$

such that for every $x \in E$ the mapping $y \mapsto r_{\alpha}(x, y)$ is $\alpha$-excessive and

$$
R_{\alpha} f(x)=\int_{E} f(y) r_{\alpha}(x, y) m(d y), \quad x \in E .
$$

It is also clear that by symmetry of $\mathbb{X}, r_{\alpha}(x, y)=r_{\alpha}(y, x)$ for $x, y \in E, \alpha \geq 0$. In what follows we put $r(x, y)=r_{0}(x, y), x, y \in E$. Thanks to the existence of $r_{\alpha}$ we may define $R_{\alpha} \mu$ for arbitrary positive Borel measure $\mu$ by putting

$$
R_{\alpha} \mu(x)=\int_{E} r_{\alpha}(x, y) \mu(d y) .
$$


It is well known (see [12, Section 5.1] and [3, Theorem V.2.1] that for each $\mu \in S$ there exists a unique perfect positive continuous additive functional $A^{\mu}$ in the Revuz duality with $\mu$, and moreover,

$$
\left(R_{\alpha} \mu\right)(x)=E_{x} \int_{0}^{\zeta} e^{-\alpha t} d A_{t}^{\mu}, \quad x \in E .
$$

\section{Linear equations}

In this section we give some definitions of a solution of the linear problem

$$
-A u=\mu,
$$

where $\mu$ is a Borel measure such that $R|\mu|(x)<\infty$ for q.e. $x \in E$. The class of such measures will be denoted by $\mathbb{M}$.

In the whole paper we adopt the convention that $\int_{E} r(x, y) d \mu(y)=0$ for every Borel measure $\mu$ on $E$ such that $\int_{E} r(x, y) d \mu^{+}(y)=\int_{E} r(x, y) d \mu^{-}(y)=\infty$. We call $u: E \rightarrow$ $\mathbb{R} \cup\{-\infty, \infty\}$ a numerical function on $E$.

\subsection{Solutions defined via the resolvent kernel and regularity results}

Definition 3.1 We say that a measurable numerical function $u$ on $E$ is a solution of (3.1) if

$$
u(x)=\int_{E} r(x, y) d \mu(y) \text { for q.e. } x \in E .
$$

Let us note that by [3, Proposition V.1.4], if the above equality holds for every $x \in E$, then $u$ is Borel measurable. Since $\mu \in \mathbb{M}, u$ is finite q.e.

Proposition 3.2 $\mathcal{M}_{b} \subset \mathbb{M}$.

Proof Since the form $\mathcal{E}$ is assumed to be transient, there exists a strictly positive Borel function $f$ on $E$ such that $R f<\infty$, q.e. From this we conclude that $f \cdot m$ is a smooth measure. Hence, by [12, Theorem 2.2.4], there exists an increasing sequence $\left\{F_{n}\right\}$ of closed subsets of $E$ such that $\bigcup_{n \geq 1} F_{n}=E$, q.e. and $\sup _{x \in E} R\left(\mathbf{1}_{F_{n}} f\right)(x)<\infty$ (see also comments following [12, Corollary 2.2.2]). As a matter of fact, in [12] in the last condition sup is replaced by ess sup with respect to $m$, however in view of [3, Proposition II.3.2], it holds true also with supremum norm. We have

$$
\left(R|\mu|, \mathbf{1}_{F_{n}} f\right) \leq\left(|\mu|, R\left(\mathbf{1}_{F_{n}} f\right)\right) \leq\|\mu\|_{T V} \cdot\left\|R\left(\mathbf{1}_{F_{n}} f\right)\right\|_{\infty} .
$$

Hence $R|\mu|$ is finite q.e., i.e. $\mu \in \mathbb{M}$.

Using Definition 3.1 we can easily prove some regularity result for solutions of (3.1). For this purpose, for $k \geq 0$ set

$$
T_{k}(u)=\max \{\min \{u, k\},-k\}, \quad u \in \mathbb{R} .
$$

Theorem 3.3 Let $\mu \in \mathcal{M}_{b}(E)$ and let $u$ be a solution of (3.1). Then $T_{k}(u) \in D_{e}[\mathcal{E}]$ and for every $k \geq 0$,

$$
\mathcal{E}\left(T_{k}(u), T_{k}(u)\right) \leq k\|\mu\|_{T V} .
$$


Proof For $\alpha \geq 0$ and measurable functions $u, v$ on $E$ set

$$
\mathcal{E}^{(\alpha)}(u, v) \equiv \alpha\left(u-\alpha R_{\alpha} u, v\right)
$$

whenever the integral exists. By the definition of a solution of (3.1), $u \cdot m=\mu \circ R$. Hence

$$
\left(R_{\alpha} u, \eta\right)=\left(u \cdot m, R_{\alpha} \eta\right)=\left(\mu \circ R, R_{\alpha} \eta\right)=\left(\mu, R R_{\alpha} \eta\right) .
$$

Therefore

$$
\begin{aligned}
\mathcal{E}^{(\alpha)}\left(u, T_{k}(u)\right) & =\alpha\left(\mu, R T_{k}(u)-\alpha R_{\alpha} R T_{k}(u)\right)=\alpha\left(\mu, R T_{k}(u)-\left(R T_{k}(u)-R_{\alpha} T_{k}(u)\right)\right) \\
& =\left(\mu, \alpha R_{\alpha} T_{k}(u)\right) \leq k\|\mu\|_{T V} .
\end{aligned}
$$

On the other hand, since $\alpha R_{\alpha}$ is Markovian, we have

$$
\mathcal{E}^{(\alpha)}\left(T_{k}(u), T_{k}(u)\right) \leq \mathcal{E}^{(\alpha)}\left(u, T_{k}(u)\right) .
$$

Consequently,

$$
\sup _{\alpha \geq 0} \mathcal{E}^{(\alpha)}\left(T_{k}(u), T_{k}(u)\right) \leq k\|\mu\|_{T V}
$$

so applying [23, Lemma I.2.11(ii)] we get the desired result.

Remark 3.4 (i) By Theorem 3.3, $T_{k}(u) \in D[\mathcal{E}]$ if $m(E)<\infty$, because by [12, Theorem 1.5.2(iii) $], D[\mathcal{E}]=D_{e}[\mathcal{E}] \cap L^{2}(E ; m)$.

(ii) $T_{k}(u) \in D[\mathcal{E}]$ if the form satisfies Poincaré type inequality $c(u, u) \leq \mathcal{E}(u, u)$ for every $u \in D[\mathcal{E}]$ and some $c>0$, because then $D_{e}[\mathcal{E}]=D[\mathcal{E}]$.

\subsection{Probabilistic solutions}

In this subsection we give an equivalent definition of solution of (3.1) using stochastic equations involving a Hunt process $\mathbb{X}$ associated with the Dirichlet operator $A$. We begin with the following lemma.

Lemma 3.5 Assume that $\mu, v \in \mathbb{M}$ and there is $\alpha_{0} \geq 0$ such that $R_{\alpha} \mu \geq R_{\alpha} v$ for $\alpha \geq \alpha_{0}$. Then $\mu \geq v$.

Proof Since $\mu, v \in \mathbb{M}$, there exists a strictly positive Borel function $\psi$ on $E$ such that $(R \psi,|\mu|+|\nu|)<\infty$. So, it is clear that it is enough to prove that $(\eta R \psi, \mu) \geq(\eta R \psi, \nu)$ for every $\eta \in C_{b}^{+}(E)$. Let $\eta \in C_{b}^{+}(E)$. An elementary calculus shows that $\alpha R_{\alpha}(\eta R \psi)(x) \rightarrow$ $\eta R \psi(x)$ for every $x \in E$. On the other hand, $\alpha R_{\alpha}(\eta R \psi)(x) \leq\|\eta\|_{\infty} R \psi(x), x \in E$. Hence, by the Lebesgue dominated convergence theorem,

$(\eta R \psi, \mu)=\lim _{\alpha \rightarrow \infty}\left(\alpha R_{\alpha}(\eta R \psi), \mu\right)=\lim _{\alpha \rightarrow \infty}\left(\eta R \psi, \alpha R_{\alpha} \mu\right) \geq \lim _{\alpha \rightarrow \infty}\left(\eta R \psi, \alpha R_{\alpha} \nu\right)=(\eta R \psi, v)$, which completes the proof.

Theorem 3.6 Assume that $\mu \in \mathbb{M}^{+}$and $\mu \perp$ Cap. Then $u=R \mu$ is quasi-continuous and the process $[0, \infty) \ni t \mapsto u\left(X_{t}\right)$ is a cádlág local martingale under the measure $P_{x}$ for q.e. $x \in E$.

Proof Let $u_{\alpha}=\alpha R_{\alpha} u, \alpha>0$. Then

$$
u_{\alpha}(x)=\alpha E_{x} \int_{0}^{\zeta} e^{-\alpha r} u\left(X_{r}\right) d r, \quad x \in E .
$$


By the Markov property, for every $t \geq 0$ and $x \in E$ we have

$$
u_{\alpha}\left(X_{t}\right)=\alpha E_{x}\left(\int_{t}^{\zeta} e^{-\alpha(r-t)} u\left(X_{r}\right) d r \mid \mathcal{F}_{t}\right), \quad P_{x} \text {-a.s. }
$$

By [12, Theorem A.2.5] the processes $t \mapsto u_{\alpha}\left(X_{t}\right), t \mapsto u\left(X_{t}\right)$ are cádlág under the measure $P_{x}$ for every $x \in E$, while by [12, Theorem 4.6.1], $u$ is quasi-continuous. Let us put

$$
\bar{N}_{t}^{\alpha, x}=\alpha E_{x}\left(\int_{0}^{\zeta} e^{-\alpha r} u\left(X_{r}\right) d r \mid \mathcal{F}_{t}\right)-u_{\alpha}\left(X_{0}\right), \quad t \geq 0,
$$

and let $N^{\alpha, x}$ denote a cádlág modification of the martingale $\bar{N}^{\alpha, x}$. Then for every $x \in E$,

$$
e^{-\alpha t} u_{\alpha}\left(X_{t}\right)=u_{\alpha}\left(X_{0}\right)-\alpha \int_{0}^{t} e^{-\alpha r} u\left(X_{r}\right) d r+\int_{0}^{t} d N_{r}^{\alpha, x}, \quad t \geq 0, \quad P_{x} \text {-a.s. }
$$

By the integration by parts formula applied to the processes $e^{\alpha t}$ and $e^{-\alpha t} u_{\alpha}(X)$ we get

$$
u_{\alpha}\left(X_{t}\right)=u_{\alpha}\left(X_{0}\right)-\int_{0}^{t} d A_{r}^{\alpha}+\int_{0}^{t} d M_{r}^{\alpha, x}, \quad t \geq 0, \quad P_{x} \text {-a.s. }
$$

where

$$
M_{t}^{\alpha, x}=\int_{0}^{t} e^{-\alpha r} d N_{r}^{\alpha, x}, \quad A_{t}^{\alpha}=\alpha \int_{0}^{t}\left(u-u_{\alpha}\right)\left(X_{r}\right) d r, \quad t \geq 0 .
$$

Since $u$ is an excessive function, $A^{\alpha}$ is an increasing process and $u_{\alpha}(x) \nearrow u(x)$ for every $x \in E$ as $\alpha \nearrow \infty$. Hence

$$
u_{\alpha}\left(X_{t}\right) \nearrow u\left(X_{t}\right), \quad t \geq 0, \quad u_{\alpha}\left(X_{t-}\right) \nearrow u\left(X_{t-}\right), \quad t>0 .
$$

Let $\left[u_{\alpha}(X)\right],[u(X)]$ denote the quadratic variations of processes $u_{\alpha}(X)$ and $u(X)$, respectively. By [12, Theorem 4.2.2] there exists an exceptional set $N \subset E$ such that for every $x \in E \backslash N$,

$$
\left[u_{\alpha}(X)\right]_{t-}=u_{\alpha}\left(X_{t-}\right), \quad[u(X)]_{t-}=u\left(X_{t-}\right), \quad t \in(0, \zeta), \quad P_{x} \text {-a.s. }
$$

Let $\zeta_{i}, \zeta_{p}$ denote the totally inaccessible and the predictable part of $\zeta$, respectively. From [12, Theorem 4.2.2] it also follows that

$$
\left[u_{\alpha}(X)\right]_{\zeta_{i}-}=u_{\alpha}\left(X_{\zeta_{i}-}\right), \quad[u(X)]_{\zeta_{i}-}=u\left(X_{\zeta_{i}-}\right), \quad P_{X} \text {-a.s. }
$$

while by the fact that $u_{\alpha}, u$ are potentials,

$$
\left[u_{\alpha}(X)\right]_{\zeta_{p-}}=[u(X)]_{\zeta_{p-}}=0 .
$$

By what has already been proved,

$$
u_{\alpha}\left(X_{t}\right) \nearrow u\left(X_{t}\right), \quad t \geq 0, \quad\left[u_{\alpha}(X)\right]_{t-} \nearrow[u(X)]_{t-}, \quad t>0 .
$$

By the generalized Dini theorem (see [10, p. 185]), $u_{\alpha}(X) \nearrow u(X)$ uniformly on compact subsets of $[0, \infty)$. Observe that for every $t \geq 0$ and q.e. $x \in E$,

$$
E_{x} u\left(X_{t}\right) \leq \liminf _{\alpha \rightarrow \infty} E_{x} u_{\alpha}\left(X_{t}\right) \leq \liminf _{\alpha \rightarrow \infty} E_{x} u_{\alpha}\left(X_{0}\right)=u(x) .
$$

Hence $u(X)$ is a supermartingale and $\lim _{t \rightarrow \infty} E_{x} u\left(X_{t}\right)<\infty$. Therefore by [25, Theorem III.13], for q.e. $x \in E$ there exists an increasing predictable process $C^{x}$ with $E_{x} C_{\zeta}^{x}<\infty$ and a cádlág local martingale $M^{x}$ such that

$$
u\left(X_{t}\right)=u\left(X_{0}\right)-C_{t}^{x}+M_{t}^{x}, \quad t \geq 0, \quad P_{x} \text {-a.s. }
$$


Since the filtration is quasi-left continuous, $M^{x}$ has no predictable jumps. Since $X$ is quasileft continuous, it also has no predictable jumps, which implies that $u(X)$ has no predictable jumps, because $u$ is quasi-continuous. Thus $C^{x}$ is continuous. Since $u(X)$ is a special semimartingale, there exists a localizing sequence $\left\{\tau_{n}^{x}\right\} \subset \mathcal{T}$ such that for every $n \geq 1$,

$$
E_{x} \sup _{t \leq \tau_{n}^{x}}\left|u\left(X_{t}\right)\right|<\infty .
$$

By [16, Proposition 3.2], $\left\{u(X)-u_{\alpha}(X)\right\}$ satisfies the so-called condition UT. Therefore by [16, Corollary 2.8], $\left[u(X)-u_{\alpha}(X)\right]_{t} \rightarrow 0$ in probability $P_{x}$ for every $t \geq 0$. But $\left[u(X)-u_{\alpha}(X)\right]=\left[M^{x}-M^{\alpha, x}\right]$. Hence $\left[M^{x}-M^{\alpha, x}\right]_{t} \rightarrow 0$ in probability $P_{x}$, which due to (3.2) is equivalent to the convergence of $\left\{M^{\alpha, x}\right\}$ to $M^{x}$ in ucp (uniform on compacts in probability). Since $u_{\alpha}(X) \rightarrow u(X)$ in ucp, $A^{\alpha} \rightarrow C^{x}$ in ucp. In fact, by (3.2), for every $n \geq 1$ we have

$$
E_{x} \sup _{t \leq \tau_{n}^{x}}\left|A_{t}^{\alpha}-C_{t}^{x}\right| \rightarrow 0 .
$$

By [12, Lemma A.3.3] there exists a process $A$ such that $A=C^{x}$ for q.e. $x \in E$. Of course, $A$ is a positive continuous additive functional. Putting

$$
M_{t}=u\left(X_{t}\right)-u\left(X_{0}\right)+A_{t}, \quad t \geq 0,
$$

we see that $M$ is an additive functional and $M^{x}=M, P_{x}$-a.s. for q.e. $x \in E$. Thus $M$ is a local martingale additive functional. By [12, Theorem 5.1.4] there exists $v \in S$ such that $A=A^{v}$. In particular, for every $\alpha \geq 0$,

$$
R_{\alpha} v(x)=E_{x} \int_{0}^{\zeta} e^{-\alpha t} d A_{t}^{v}
$$

for q.e. $x \in E$. Observe that by the resolvent identity, for every $\alpha \geq 0$ we have

$$
u=R_{\alpha}(\mu+\alpha u) .
$$

On the other hand, by (3.2) and the integration by parts formula applied to the processes $e^{-\alpha t}$ and $u\left(X_{t}\right)$,

$$
u(x)=E_{x} e^{-\alpha \tau_{k}^{x}} u\left(X_{\tau_{k}^{x}}\right)+E_{x} \int_{0}^{\tau_{k}^{x}} e^{-\alpha r} d A_{r}^{v}+\alpha E_{x} \int_{0}^{\tau_{k}^{x}} e^{-\alpha r} u\left(X_{r}\right) d r .
$$

It is clear that

$$
E_{x} \int_{0}^{\tau_{k}^{x}} e^{-\alpha r} d A_{r}^{v} \rightarrow R_{\alpha} v(x), \quad \alpha E_{x} \int_{0}^{\tau_{k}^{x}} e^{-\alpha r} u\left(X_{r}\right) d r \rightarrow \alpha R_{\alpha} u(x)
$$

as $k \rightarrow \infty$. From this, (3.4) and (3.5) we conclude that for q.e $x \in E$,

$$
\lim _{k \rightarrow \infty} E_{x} e^{-\alpha \tau_{k}^{x}} u\left(X_{\tau_{k}^{x}}\right)=R_{\alpha}(\mu-v)(x) .
$$

By this and [3, Proposition II.3.2], $R_{\alpha}(\mu-v) \geq 0$. Since $\alpha \geq 0$ was arbitrary, applying Lemma 3.5 shows that $\mu \geq v$. Since $\mu \perp$ Cap, it follows that $v \equiv 0$ or, equivalently, that $A^{v} \equiv 0$. Therefore from (3.3) it follows that $u(X)$ is a local martingale.

Let us recall that a process $M$ is called a local martingale additive functional (MAF) if it is an additive functional and $M$ is an $\left(\mathcal{F}, P_{x}\right)$-local martingale for q.e. $x \in E$. 
Theorem 3.7 Assume that $\mu \in \mathbb{M}^{+}$and let $u=R \mu$. Then $u$ is quasi-continuous and there exists a local MAF $M$ such that

$$
u\left(X_{t}\right)=u\left(X_{0}\right)-\int_{0}^{t} d A_{r}^{\mu_{d}}+\int_{0}^{t} d M_{r}, \quad t \geq 0, \quad P_{x} \text {-a.s. }
$$

for q.e. $x \in E$. Moreover, for every polar set $N \subset E$, every stopping time $T \geq \zeta$ and sequence $\left\{\tau_{k}\right\} \subset \mathcal{T}$ such that $\tau_{k} \nearrow T$ and $E_{x} \sup _{t \leq \tau_{k}} u\left(X_{t}\right)<\infty$ for $x \in E \backslash N$ and $k \geq 1$ we have

$$
\lim _{k \rightarrow \infty} E_{x} u\left(X_{\tau_{k}}\right)=R \mu_{c}(x), \quad x \in E \backslash N .
$$

Proof Let $w=R \mu_{c}$ and $v=R \mu_{d}$. It is well known (see [17, Lemma4.3]) that $v$ is quasicontinuous and that there exists a uniformly integrable MAF $M^{v}$ such that

$$
v\left(X_{t}\right)=v\left(X_{0}\right)-\int_{0}^{t} d A_{r}^{\mu_{d}}+\int_{0}^{t} d M_{r}^{v}, \quad t \geq 0, \quad P_{x} \text {-a.s. }
$$

for q.e. $x \in E$. By Theorem 3.6, $w$ is quasi-continuous and there exists a local MAF $M^{w}$ such that

$$
w\left(X_{t}\right)=w\left(X_{0}\right)+\int_{0}^{t} d M_{r}^{w}, \quad t \geq 0, \quad P_{x} \text {-a.s. }
$$

for q.e. $x \in E$. Let $N \subset E$ be a polar set such that (3.8), (3.9) hold for $x \in E \backslash N$. Let $\left\{\tau_{k}\right\}$ be as in the formulation of the theorem. Then $M^{v, \tau_{k}}, M^{w, \tau_{k}}$ are both uniformly integrable and by (3.8) and (3.9),

$$
u(x)=E_{x} u\left(X_{\tau_{k}}\right)+E_{x} \int_{0}^{\tau_{k}} d A_{r}^{\mu_{d}}, \quad x \in E \backslash N .
$$

Letting $k \rightarrow \infty$ in the above equation yields

$$
R \mu(x)=u(x)=\lim _{k \rightarrow \infty} E_{x} u\left(X_{\tau_{k}}\right)+R \mu_{d}(x), \quad x \in E \backslash N,
$$

which proves (3.7). Adding (3.8) to (3.9) gives (3.6).

Remark 3.8 Under the assumptions of Theorem 3.7, for every $\alpha>0$,

$$
\lim _{k \rightarrow \infty} E_{x} e^{-\alpha \tau_{k}} u\left(X_{\tau_{k}}\right)=R_{\alpha} \mu_{c}(x), \quad x \in E \backslash N .
$$

To see this we use (3.4) and arguments following it.

We are now ready to introduce the second definition of a solution of (3.1) making use of the Hunt process $\mathbb{X}$ associated with operator $A$. Solutions of (3.1) in the sense of this definition will be called probabilistic solutions or simply solutions, because we will show that our second definition is equivalent to the definition via the resolvent kernel.

Definition 3.9 We say that a measurable numerical function $u$ on $E$ is a probabilistic solution of (3.1) if

(a) there exists a local MAF $M$ such that for q.e. $x \in E$,

$$
u\left(X_{t}\right)=u\left(X_{0}\right)-\int_{0}^{t} d A_{r}^{\mu_{d}}+\int_{0}^{t} d M_{r}, \quad t \geq 0, \quad P_{x} \text {-a.s. }
$$

(b) for every polar set $N \subset E$, every stopping time $T \geq \zeta$ and every sequence $\left\{\tau_{k}\right\} \subset \mathcal{T}$ such that $\tau_{k} \nearrow T$ and $E_{x} \sup _{t \leq \tau_{k}}\left|u\left(X_{t}\right)\right|<\infty$ for every $x \in E \backslash N$ and $k \geq 1$ we have

$$
E_{x} u\left(X_{\tau_{k}}\right) \rightarrow R \mu_{c}(x), \quad x \in E \backslash N .
$$


Any sequence $\left\{\tau_{k}\right\}$ with the properties listed in (b) will be called the reducing sequence for $u$, and we will say that $\left\{\tau_{k}\right\}$ reduces $u$.

Remark 3.10 Since $u(X)$ in the above definition is a special semimartingale, there exists at least one reducing sequence $\left\{\tau_{k}\right\}$ for $u$. In fact, the stopping times defined as

$$
\tau_{k}=\inf \left\{t \geq 0 ;\left|u\left(X_{t}\right)\right| \geq k\right\} \wedge k, \quad k \geq 1
$$

form a reducing sequence (see the reasoning in the proof of [26, Theorem 51.1]).

Remark 3.11 If $\mu$ is a smooth measure then Definition 3.9 reduces to the definition of a solution introduced in [17]. Indeed, by condition (a),

$$
u(x)=E_{x} u\left(X_{\tau_{k}}\right)+E_{x} \int_{0}^{\tau_{k}} d A_{r}^{\mu_{d}}
$$

for q.e. $x \in E$. Therefore letting $k \rightarrow \infty$ and using (b) we see that for q.e. $x \in E$,

$$
u(x)=E_{x} \int_{0}^{\zeta} d A_{r}^{\mu_{d}} .
$$

Note that if $A$ is a uniformly elliptic divergence form operator then by [17, Proposition 5.3], $u$ is also a solution of (3.1) in the sense of Stampacchia (see [27]). In the sequel we will show that this holds true for general Borel measures and wider class of operators.

Proposition 3.12 A measurable function $u$ on $E$ is a probabilistic solution of (3.1)if and only if it is a solution of (3.1) in the sense of Definition 3.1.

Proof Assume that $u$ is a solution of (3.1) in the sense of Definition 3.1. Then by Theorem 3.7, $u$ is a probabilistic solution. Now suppose that $u$ is a probabilistic solution of (3.1). Then using (a) and (b) of the definition of a probabilistic solution of (3.1) we obtain

$$
u(x)=R \mu_{c}(x)+E_{x} \int_{0}^{\zeta} d A_{r}^{\mu_{d}}=R \mu(x)=\int_{E} r(x, y) \mu(d y)
$$

for q.e. $x \in E$.

\section{Semilinear equations}

In what follows $\mu \in \mathbb{M}$ and $f: E \times \mathbb{R} \rightarrow \mathbb{R}$ is a function satisfying the following conditions: $\mathbb{R} \ni y \mapsto f(x, y)$ is continuous for every $x \in E$ and $E \ni x \mapsto f(x, y)$ is measurable for every $y \in \mathbb{R}$.

In this section we consider semilinear equation of the form

$$
-A u=f(x, u)+\mu \text {. }
$$

Definition 4.1 We say that a measurable numerical function $u$ on $E$ is a solution of (4.1) if $f(\cdot, u) \cdot m \in \mathbb{M}$ and $u$ is a solution of (3.1) with $\mu$ replaced by $f(\cdot, u) \cdot m+\mu$.

We will need the following hypotheses:

(H1) for every $x \in E$ the mapping $y \mapsto f(x, y)$ in nonincreasing,

(H2) for every $y \in \mathbb{R}$ the mapping $x \mapsto f(x, y) \in q L^{1}(E ; m)$,

(H3) $f(\cdot, 0) \cdot m \in \mathbb{M}$. 


\subsection{Comparison results, a priori estimates and regularity of solutions}

In the sequel, for a given real function $u$ on $E$ we write

$$
f_{u}(x)=f(x, u(x)), \quad x \in E .
$$

Proposition 4.2 Assume that $\mu_{1}, \mu_{2} \in \mathbb{M}, \mu_{1} \leq \mu_{2}, f^{1}(x, y) \leq f^{2}(x, y)$ for $x \in E, y \in \mathbb{R}$ and $f^{1}$ or $f^{2}$ satisfies (H1). Then $u_{1} \leq u_{2}$ q.e., where $u_{1}$ (resp. $u_{2}$ ) is a solution of (4.1) with data $f^{1}, \mu^{1}$ (resp. $\left.f^{2}, \mu^{2}\right)$.

Proof Let $\left\{\tau_{k}\right\}$ be a common reducing sequence for $u_{1}$ and $u_{2}$. We assume that $f^{1}$ satisfies (H1). By the Tanaka-Meyer formula (see [25, Theorem IV.66]), for every $k \geq 1$,

$$
\begin{aligned}
\left(u_{1}(x)-u_{2}(x)\right)^{+} \leq & E_{x}\left(u_{1}-u_{2}\right)^{+}\left(X_{\tau_{k}}\right)+E_{x} \int_{0}^{\tau_{k}} \mathbf{1}_{\left\{u_{1}>u_{2}\right\}}\left(X_{r}\right)\left(f_{u_{1}}^{1}-f_{u_{2}}^{2}\right)\left(X_{r}\right) d r \\
& +E_{x} \int_{0}^{\tau_{k}} \mathbf{1}_{\left\{u_{1}>u_{2}\right\}}\left(X_{r}\right) d\left(A_{r}^{\mu_{d}^{1}}-A_{r}^{\mu_{d}^{2}}\right)
\end{aligned}
$$

for q.e. $x \in E$. From the assumption $\mu_{1} \leq \mu_{2}$ and properties of the Revuz duality it follows that $d A^{\mu_{d}^{1}} \leq d A^{\mu_{d}^{2}}, P_{x}$-a.s. for q.e. $x \in E$. By (H1) and the assumptions on $f^{1}$ and $f^{2}$,

$$
\mathbf{1}_{\left\{u_{1}>u_{2}\right\}}\left(f_{u_{1}}^{1}-f_{u_{2}}^{2}\right)=\mathbf{1}_{\left\{u_{1}>u_{2}\right\}}\left(f_{u_{1}}^{1}-f_{u_{2}}^{1}\right)+\mathbf{1}_{\left\{u_{1}>u_{2}\right\}}\left(f_{u_{2}}^{1}-f_{u_{2}}^{2}\right) \leq 0 .
$$

Hence

$$
\left(u_{1}(x)-u_{2}(x)\right)^{+} \leq E_{x}\left(u_{1}-u_{2}\right)^{+}\left(X_{\tau_{k}}\right), \quad k \geq 1
$$

for q.e. $x \in E$. But

$$
\left(u_{1}-u_{2}\right)^{+}=\left(R\left(f_{u_{1}}^{1}+\mu^{1}-\mu^{2}-f_{u_{2}}^{2}\right)\right)^{+} \leq R\left(f_{u_{1}}^{1}+\mu^{1}-\mu^{2}-f_{u_{2}}^{2}\right)^{+} .
$$

Therefore

$$
\left(u_{1}(x)-u_{2}(x)\right)^{+} \leq \limsup _{k \rightarrow \infty} E_{x}\left(u_{1}-u_{2}\right)^{+}\left(X_{\tau_{k}}\right) \leq R\left(\mu_{c}^{1}-\mu_{c}^{2}\right)^{+}=0
$$

for q.e. $x \in E$, which proves the proposition.

Corollary 4.3 Under (H1) there exists at most one solution of (4.1).

Proposition 4.4 Let $u_{1}, u_{2}$ be solutions of (4.1) with $\mu_{1} \in \mathbb{M}$ and $\mu_{2} \in \mathbb{M}$, respectively. If $f$ satisfies (H1), then

$$
R\left|f_{u_{1}}-f_{u_{2}}\right|(x) \leq R\left|\mu_{1}-\mu_{2}\right|(x), \quad x \in E .
$$

Proof Let $\left\{\tau_{k}\right\}$ be a common reducing sequence for $u_{1}$ and $u_{2}$. By the Tanaka-Meyer formula,

$$
\begin{aligned}
\left|u_{1}(x)-u_{2}(x)\right| \leq & E_{x}\left|u_{1}-u_{2}\right|\left(X_{\tau_{k}}\right)+E_{x} \int_{0}^{\tau_{k}} \operatorname{sgn}\left(u_{1}-u_{2}\right)\left(X_{r}\right)\left(f_{u_{1}}-f_{u_{2}}\right)\left(X_{r}\right) d r \\
& +E_{x} \int_{0}^{\tau_{k}} \operatorname{sgn}\left(u_{1}-u_{2}\right)\left(X_{r}\right) d\left(A_{r}^{\mu_{d}^{1}}-A_{r}^{\mu_{d}^{2}}\right)
\end{aligned}
$$

for q.e. $x \in E$. By (H1) the second term on the right-hand side of (4.2) is nonpositive. Therefore from (4.2) it follows that

$$
E_{x} \int_{0}^{\tau_{k}}\left|f_{u_{1}}-f_{u_{2}}\right|\left(X_{r}\right) d r \leq E_{x}\left|u_{1}-u_{2}\right|\left(X_{\tau_{k}}\right)+E_{x} \int_{0}^{\tau_{k}} d A_{r}^{\left|\mu_{d}^{1}-\mu_{d}^{2}\right|}
$$


for q.e $x \in E$. Letting $k \rightarrow \infty$ we get

$$
E_{x} \int_{0}^{\zeta}\left|f_{u_{1}}-f_{u_{2}}\right|\left(X_{r}\right) d r \leq R\left|\mu_{c}^{1}-\mu_{c}^{2}\right|(x)+R\left|\mu_{d}^{1}-\mu_{d}^{2}\right|(x)=R\left|\mu_{1}-\mu_{2}\right|(x)
$$

for q.e. $x \in E$ (see the reasoning at the end of the proof of Proposition 4.2). From this and [3, Proposition II.3.2] we get the desired result.

Proposition 4.5 Let $u$ be a solution of (4.1) with $f$ satisfying (H1), (H3). Then

$$
R\left|f_{u}\right|(x) \leq 2 R|f(\cdot, 0)|(x)+R|\mu|(x), \quad x \in E .
$$

Proof We apply Proposition 4.4 to $u_{1}=u, u_{2}=0, \mu_{1}=\mu, \mu_{2}=-f(\cdot, 0)$.

Given a positive function $\rho \in \mathcal{S}$, we denote by $\mathcal{M}_{\rho}$ the set of all measures $\mu \in \mathcal{M}$ such that $\|\mu\|_{\rho}<\infty$, where $\|\mu\|_{\rho}=\|\rho \cdot \mu\|_{T V}$.

Important examples of positive $\rho \in \mathcal{S}$ are $\rho=1$ and $\rho=R \eta$, where $\eta$ is a positive Borel function on $E$. Let us also note that if $A=\Delta^{\alpha}$ (with $\alpha \in(0,1]$ ) on an open bounded set $D \subset \mathbb{R}^{d}$ (see Remark 4.13) then for $\rho=R 1$ we have $\mathcal{M}_{\rho}=\left\{\mu \in \mathcal{M}: \delta^{\alpha} \cdot \mu \in \mathcal{M}_{b}\right\}$, where $\delta(x)=\operatorname{dist}(x, \partial D)$, because by [22] there exists $c, C>0$ such that

$$
c \delta^{\alpha}(x) \leq R 1(x) \leq C \delta^{\alpha}(x), \quad x \in D .
$$

In the rest of the paper we assume that $\rho \in \mathcal{S}$ and $\rho$ is strictly positive.

Lemma 4.6 Assume that $\mu, v \in \mathcal{M}_{\rho}$ and $R \mu(x) \leq R v(x)$ for $x \in E$. Then $\|\mu\|_{\rho} \leq\|v\|_{\rho}$.

Proof By [3, Proposition II.2.6] there exists a sequence $\left\{h_{n}\right\}$ of positive bounded Borel functions on $E$ such that $R h_{n} \nearrow \rho$. For $n \geq$ we have

$$
\left(\mu, R h_{n}\right)=\left(R \mu, h_{n}\right) \leq\left(R v, h_{n}\right)=\left(v, R h_{n}\right),
$$

so letting $n \rightarrow \infty$ we get the desired result.

Proposition 4.7 Let $u_{1}, u_{2}$ be solutions of (4.1) with $\mu_{1} \in \mathcal{M}_{\rho}$ and $\mu_{2} \in \mathcal{M}_{\rho}$, respectively. If $f$ satisfies (H1) then

$$
\left\|f_{u_{1}}-f_{u_{2}}\right\|_{L^{1}(E ; \rho \cdot m)} \leq\left\|\mu_{1}-\mu_{2}\right\|_{\rho}
$$

Proof Follows from Proposition 4.4 and Lemma 4.6.

Proposition 4.8 Let $u$ be a solution of (4.1) with $\mu \in \mathcal{M}_{\rho}$ and $f$ satisfying (H1) and such that $f(\cdot, 0) \in L^{1}(E ; \rho \cdot m)$. Then

$$
\left\|f_{u}\right\|_{L^{1}(E ; \rho \cdot m)} \leq 2\|f(\cdot, 0)\|_{L^{1}(E ; \rho \cdot m)}+\|\mu\|_{\rho} .
$$

Proof Follows from Proposition 4.5 and Lemma 4.6.

Theorem 4.9 Let $u$ be a solution of (4.1) with $\mu \in \mathcal{M}_{b}$ and $f$ satisfying (H1) and such that $f(\cdot, 0) \in L^{1}(E ; m)$. Then for every $k \geq 0, T_{k}(u) \in D_{e}[\mathcal{E}]$ and

$$
\mathcal{E}\left(T_{k}(u), T_{k}(u)\right) \leq 2 k\left(\|f(\cdot, 0)\|_{L^{1}}+\|\mu\|_{T V}\right) .
$$

Proof Follows from Theorem 3.3 and Proposition 4.8. 


\subsection{Stampacchia's definition by duality}

In [27] Stampacchia introduced a definition of a solution of (3.1) in case $\mu \in \mathcal{M}_{b}$ and $A$ is uniformly elliptic operator of the form

$$
A=\sum_{i, j=1}^{d} \frac{\partial}{\partial x_{j}}\left(a_{i j} \frac{\partial}{\partial x_{i}}\right)
$$

on a bounded open set $D \subset \mathbb{R}^{d}$. According to this definition, now called Stampacchia's definition by duality, a measurable function $u \in L^{1}(D ; m)$, where $m$ is the Lebesgue measure on $\mathbb{R}^{d}$, is a solution of (3.1) if

$$
(u, \eta)=(G \eta, \mu), \quad \eta \in L^{\infty}(D ; m) .
$$

The above definition has sense, because it is well known that for $A$ as above $G \eta$ has a bounded continuous version. In the general case considered in the paper the original Stampacchia's definition has to be modified, because the measure $\mu$ is not assumed to be bounded, $G \eta$ may be not continuous for $\eta \in L^{\infty}(E ; m)$ and moreover, the solution of (3.1) may be not locally integrable (see [17, Example 5.7]). In [17] we introduced a generalized Stampacchia's definition for solutions of (4.1) with Dirichlet operator $A$ and bounded measure $\mu$ such that $\mu \ll$ Cap. Here we give a definition for general measures of the class $\mathbb{M}$.

Lemma 4.10 We have $\mathbb{M}=\bigcup \mathcal{M}_{\rho}$, where the union is taken over all strictly positive excessive bounded functions.

Proof It is clear that $\bigcup \mathcal{M}_{\rho} \subset \mathbb{M}$. To prove the opposite inclusion, let us assume that $\mu \in \mathbb{M}$. Then $R|\mu|<\infty, m$-a.e. Therefore there exists a strictly positive Borel function $\eta$ on $E$ such that $(R|\mu|, \eta)=(|\mu|, R \eta)<\infty$. On the other hand, since the form $(\mathcal{E}, D[\mathcal{E}])$ is transient, there exists a strictly positive Borel function $g$ on $E$ such that $\|R g\|_{\infty}<\infty$ (see [24, Corollary 1.3.6]). Let us put $\rho=R(\eta \wedge g)$. It is clear that $\rho$ is*** a bounded strictly positive excessive function.

Definition 4.11 We say that a measurable numerical function $u$ on $E$ is a solution of (4.1) in the sense of Stampacchia if for every $\eta \in \mathcal{B}(E)$ such that $(|\mu|, R|\eta|)<\infty$ the integrals $(u, \eta),\left(f_{u}, R \eta\right)$ are finite and we have

$$
(u, \eta)=\left(f_{u}, R \eta\right)+(\mu, R \eta) .
$$

Proposition 4.12 Let $\mu \in \mathbb{M}$. A measurable function $u$ on $E$ is a solution of (4.1) in the sense of Definition 4.11 if and only if it is a solution of (4.1) in the sense of Definition 3.1.

Proof Let $u$ be a solution of (4.1) in the sense od Definition 3.1. Then by Proposition 4.5, $|u|+R\left|f_{u}\right| \leq R|\mu|$, it is clear that $u$ is a solution of (4.1) in the sense of Stampacchia. Now assume that $u$ is a solution of (4.1) in the sense of Stampacchia. By Lemma 4.10 there exists a strictly positive $\rho \in \mathcal{S}$ such that $\mu \in \mathcal{M}_{\rho}$. In fact, from the proof of Lemma 4.10 it follows that we may take $\rho=R g$ for some strictly positive Borel function $g$ on $E$. We have

$$
\left(u, g \mathbf{1}_{B}\right)=\left(R f_{u}, g \mathbf{1}_{B}\right)+\left(R \mu, g \mathbf{1}_{B}\right)
$$

for every $B \in \mathcal{B}(E)$. Hence $u=R f_{u}+R \mu, m$-a.e., and the proof is complete. 
Remark 4.13 Let $\alpha \in(0,1]$ and let $D$ be an open subset of $\mathbb{R}^{d}$. Denote by $(\mathcal{E}, D[\mathcal{E}])$ the Dirichlet form associated with the operator $\Delta^{\alpha}$ on $\mathbb{R}^{d}$ (see [12, Example 1.4.1]), and by $\left(\mathcal{E}_{D}, D\left[\mathcal{E}_{D}\right]\right)$ the part of $(\mathcal{E}, D[\mathcal{E}])$ on $D$ (see [12, Section 4.4]). By $A$ denote the operator associated with $\left(\mathcal{E}_{D}, D\left[\mathcal{E}_{D}\right]\right)$, i.e. the fractional Laplacian $\Delta^{\alpha}$ on $D$ with zero boundary condition. If $\mu \in \mathcal{M}_{\delta}^{\alpha}$ then in Definition 4.11 one can take any function $\eta \in \mathcal{B}_{b}(E)$ as a test function. It follows in particular that in case of equations involving operator $A$ Stampacchia's definition is equivalent to the one introduced in [8, Definition 1.1].

Remark 4.14 In [18] renormalized solutions of (4.1) are defined in case $\mu$ is a bounded smooth measure. It is also proved there that $u$ is a renormalized solution of (4.1) if and only it is a probabilistic solution. Thus, in case $\mu$ is smooth, all the definitions (renormalized, Stampacchia's by duality, probabilistic, via the resolvent kernel) are equivalent.

Remark 4.15 In case $A$ is the Laplace operator on an open bounded set $D \subset \mathbb{R}^{d}$, also the socalled weak solutions of (4.1) are considered in the literature (see, e.g., [5]). A weak solution of (4.1) is a function $u \in L^{1}(D ; d x)$ such that $f_{u} \in L^{1}(D ; d x)$ and for every $\eta \in C_{0}^{\infty}(\bar{D})$,

$$
-\int_{D} u \Delta \eta d x=\int_{D} f_{u} \eta d x+\int_{D} \eta d \mu .
$$

It is clear that the definition of weak solution is equivalent to Stampacchia's definition by duality. It is worth pointing out that in fact the concept of weak solutions is also due to Stampacchia (see [27, Definition 9.1]).

\subsection{Existence of solutions}

In [17] (see also [19] for the case of operator corresponding to general nonsymmetric quasiregular form) it is proved that if $\mu$ is smooth then under conditions (H1)-(H3) there exists a solution of (4.1). It is well known that if $A=\Delta$ and $\mu$ is not smooth, i.e. $\mu_{c} \neq 0$, then in general assumptions (H1)-(H3) are not sufficient for the existence of a solution of (4.1). In this section we give an existence result for (4.1) under the following additional hypothesis:

(H4) there exists a positive Borel measurable function $g$ on $E$ such that $g \cdot m \in \mathbb{M}$ and $|f(x, y)| \leq g(x), x \in E, y \in \mathbb{R}$.

Let us observe that (H4) implies (H2), (H3). In the paper we have assumed Meyer's hypothesis (L), so we may also drop (H1).

Hypothesis (H4) imposes rather restrictive assumption on the growth of $f$ but allows us to prove the existence of solutions for any $\mu \in \mathbb{M}$ and any Dirichlet operator $A$.

Theorem 4.16 Assume (H4). Then there exists a solution of (4.1).

Proof Let $\varrho$ be a strictly positive Borel function on $E$ such that

$$
r:=\int_{E}(R g(x)+R|\mu|(x)) \varrho(x) m(d x)<\infty .
$$

Let us define $\Phi: L^{1}(E ; \varrho \cdot m) \rightarrow L^{1}(E ; \varrho \cdot m)$ by

$$
\Phi(u)=R f(\cdot, u)+R \mu .
$$

Observe that for every $u \in L^{1}(E, \varrho \cdot m),\|\Phi(u)\|_{L^{1}(E ; \varrho \cdot m)} \leq r$. It is an elementary check that $\Phi$ is continuous. Let $\left\{u_{n}\right\} \subset L^{1}(E ; \varrho \cdot m)$ and let $v_{n}=\Phi\left(u_{n}\right)$. By [11, Lemma 94, page 306], $\left\{v_{n}\right\}$ has a subsequence convergent $m$-a.e., which when combined with the fact that 
$\left|v_{n}\right|(x) \leq R g(x)+R|\mu|(x)$ for $x \in E$ implies that, up to a subsequence, $\left\{v_{n}\right\}$ converges in $L^{1}(E ; \varrho \cdot m)$. Therefore by the Schauder fixed point theorem there exists $u \in L^{1}(E ; \varrho \cdot m)$ such that $\Phi(u)=u$, which proves the theorem.

\section{Good measures and reduced measures}

In this section we develop the theory of reduced measures for (1.1) in case of general Dirichlet operator $A$ and general measure $\mu$ of the class $\mathbb{M}$. Our results generalize the corresponding results from H. Brezis, M. Marcus and A.C. Ponce [5] proved in the case where $A$ is the Laplace operator on a bounded domain in $\mathbb{R}^{d}$ and $\mu$ is a bounded measure. Also note that in [5] it is assumed that $f$ does not depend on $x$.

In the whole section in addition to (H1)-(H3) we assume that $f(x, y)=0$ for $y \leq 0$.

Definition 5.1 We say that a measurable numerical function $v$ on $E$ is a subsolution of (4.1) if $f_{v} \cdot m \in \mathbb{M}$ and there exists a measure $v \in \mathbb{M}$ such that $v \leq \mu$ and

$$
-A v=f(x, v)+v .
$$

Theorem 5.2 Assume (H1)-(H3). Let $f_{n}=f \vee(-n)$ and let $u_{n}$ be a solution of

$$
-A u_{n}=f_{n}\left(x, u_{n}\right)+\mu .
$$

Then $u_{n} \searrow u^{*}$, where $u^{*}$ is a maximal subsolution of (4.1). Moreover, the measure $\mu^{*}=$ $-A u^{*}-f\left(x, u^{*}\right)$ admits decomposition of the form $\mu^{*}=\mu_{d}+v$ with $v \perp$ Cap such that $v \leq \mu_{c}$.

Proof Let $\left\{\tau_{k}\right\}$ be a reducing sequence for $u_{n}$. By the Tanaka-Meyer formula,

$$
\begin{aligned}
\left|u_{n}(x)\right| \leq & E_{x}\left|u_{n}\right|\left(X_{\tau_{k}}\right)+E_{x} \int_{0}^{\tau_{k}} \operatorname{sgn}\left(u_{n}\right)\left(X_{r}\right) f_{n}\left(X_{r}, u_{n}\left(X_{r}\right)\right) d r \\
& +E_{x} \int_{0}^{\tau_{k}} \operatorname{sgn}\left(u_{n}\right)\left(X_{r}\right) d A_{r}^{\mu_{d}}
\end{aligned}
$$

for q.e. $x \in E$. By $(\mathrm{H} 1)$,

$$
\left|u_{n}(x)\right|+E_{x} \int_{0}^{\tau_{k}}\left|f_{n}\left(X_{r}, u_{n}\left(X_{r}\right)\right)\right| d r \leq E_{x}\left|u_{n}\right|\left(X_{\tau_{k}}\right)+E_{x} \int_{0}^{\tau_{k}} d A_{r}^{\left|\mu_{d}\right|}
$$

for q.e. $x \in E$. Letting $k \rightarrow \infty$ in the above inequality we get

$$
\left|u_{n}(x)\right|+R\left|f_{n}\left(\cdot, u_{n}\right)\right|(x) \leq R|\mu|(x)
$$

for q.e. $x \in E$. Let $v_{n}, w_{n}$ be solutions of the following equations

$$
-A v_{n}=f_{n}^{+}\left(\cdot, u_{n}\right)+\mu^{+}, \quad-A w_{n}=f_{n}^{-}\left(\cdot, u_{n}\right)+\mu^{-} .
$$

Of course, $v_{n}, w_{n}$ are excessive functions and by (5.1),

$$
v_{n}=R f_{n}^{+}\left(\cdot, u_{n}\right)+R \mu^{+} \leq 2 R|\mu|, \quad w_{n}=R f_{n}^{-}\left(\cdot, u_{n}\right)+R \mu^{-} \leq 2 R|\mu| .
$$

By [11, Lemma 94, page 306], from $\left\{v_{n}\right\}$ and $\left\{w_{n}\right\}$ one can choose subsequences convergent $m$-a.e. to excessive functions $v$ and $w$, respectively. By (5.2) and [14], there exists $v_{1}, v_{2} \in \mathbb{M}^{+}$ such that $v=R v_{1}, w=R v_{2}$. By Theorem 3.7 the function $h=R|\mu|$ is quasi-continuous. Therefore if we put $\delta_{k}^{1}=\inf \left\{t \geq 0: h\left(X_{t}\right) \geq k\right\} \wedge \zeta$, then $\delta_{k}^{1} \nearrow \zeta, P_{x}$-a.s. for q.e. $x \in E$. 
From Theorem 3.7 it also follows that $h(X)$ is a special semimartingale. Therefore there exists a sequence $\left\{\delta_{k}^{2}\right\} \subset \mathcal{T}$ such that $\delta_{k}^{2} \nearrow \zeta$ and for q.e. $x \in E$,

$$
E_{x} \sup _{t \leq \delta_{k}^{2}}\left|h\left(X_{t}\right)\right|<\infty .
$$

We may assume that $\tau_{k}=\delta_{k}^{1}=\delta_{k}^{2}$. Since by Proposition 4.2, $u_{n}(x) \geq u_{n+1}(x), n \geq 1$, for q.e. $x \in E$, there exists $u^{*}$ such that $u_{n} \searrow u^{*}$, q.e. Therefore letting $n \rightarrow \infty$ in the equation

$$
u_{n}(x)=E_{x} u_{n}\left(X_{\tau_{k}}\right)+E_{x} \int_{0}^{\tau_{k}} f_{n}\left(X_{r}, u_{n}\left(X_{r}\right)\right) d r+E_{x} \int_{0}^{\tau_{k}} d A_{r}^{\mu_{d}}
$$

and using (H1)-(H3), (5.1) (and the fact that $\tau_{k}=\delta_{k}^{1}=\delta_{k}^{2}$ ) we get

$$
u^{*}(x)=E_{x} u^{*}\left(X_{\tau_{k}}\right)+E_{x} \int_{0}^{\tau_{k}} f\left(X_{r}, u^{*}\left(X_{r}\right)\right) d r+E_{x} \int_{0}^{\tau_{k}} d A_{r}^{\mu_{d}}
$$

for q.e. $x \in E$. Observe that $u^{*}=v-w=R v$, q.e., where $v=v_{1}-v_{2}$. Therefore by Theorem 3.7,

$$
\lim _{k \rightarrow \infty} E_{x} u^{*}\left(X_{\tau_{k}}\right)=R v_{c}(x)
$$

for q.e. $x \in E$. By (5.1) and Fatou's lemma, $f\left(\cdot, u^{*}\right) \cdot m \in \mathbb{M}$. Hence $u^{*}$ is a solution of (4.1) with $\mu$ replaced by $\mu^{*}:=\mu_{d}+v_{c}$. What is left is to show that $u^{*}$ is the maximal subsolution of (4.1). By the construction of $u^{*}, u_{n} \geq u^{*}$. Therefore by condition (b) of the definition of a probabilistic solution of (4.1) and Lemma 3.5 (see also Remark 3.8) we have $\mu_{c}^{*} \leq \mu_{c}$, which when combined with the fact that $\mu_{d}^{*}=\mu_{d}$ shows that $\mu^{*} \leq \mu$, i.e. that $u^{*}$ is subsolution of (4.1). Suppose that $v$ is another subsolution of (4.1). Then there exists $\beta \in \mathbb{M}$ such that $\beta \leq \mu$ and $v$ is a solution of (4.1) with $\mu$ replaced by $\beta$. Since $\beta \leq \mu$ and $f_{n} \geq f$, applying Proposition 4.2 shows that $u_{n} \geq v$ q.e., hence that $u^{*} \geq v$ q.e., which completes the proof.

Let $\mu \in \mathbb{M}$. From now on by $\mu^{*}, u^{*}$ we denote the objects constructed in Theorem 5.2. By Theorem 5.2, $\mu^{*} \leq \mu$. It is known (see [2]) that it may happen that $\mu^{*} \neq \mu$, i.e. that there is no solution of (4.1) under assumptions (H1)-(H3).

Definition 5.3 (a) We call $\mu^{*}$ the reduced measure associated to $\mu$.

(b) We call $\mu \in \mathbb{M}$ a good measure (relative to $A$ and $f$ ) if there exists a solution of (4.1).

In what follows we denote by $\mathcal{G}$ the set of all good measures relative to $A$ and $f$. Of course, $\mu^{*} \in \mathcal{G}$.

\section{Proposition 5.4 Let $\mu \in \mathbb{M}$. Then}

(i) $\mu^{*} \leq \mu$,

(ii) $\mu-\mu^{*} \perp \operatorname{Cap},\left(\mu^{*}\right)_{d}=\mu_{d}$,

(iii) $\mathcal{A} \cap S \subset \mathcal{G}$,

(iv) $\mu^{*}$ is the largest good measure less then or equal to $\mu$,

(v) $\left|\mu^{*}\right| \leq|\mu|$,

(vi) if $\mu, v \in \mathbb{M}$ and $\mu \leq v$, then $\mu^{*} \leq v^{*}$.

Proof Assertions (i) and (ii) follow from Theorem 5.2. (iii) follows from [17]. Let $v \in \mathcal{G}$ and $v \leq \mu$. Since $v \in \mathcal{G}$, there exist a solution $v$ of (4.1) with $\mu$ replaced by $v$. Since $v \leq \mu$, the latter means that $v$ is a subsolution of (4.1). Therefore by Theorem 5.2, $v \leq u^{*}$ q.e. From this, condition (b) of the definition of a probabilistic solution and Remark 3.8,

$$
R_{\alpha} v_{c} \leq R_{\alpha}\left(\mu^{*}\right)_{c}
$$


for every $\alpha \geq 0$. Hence $v_{c} \leq\left(\mu^{*}\right)_{c}$ by Lemma 3.5. On the other hand, since $v \leq \mu, v_{d} \leq \mu_{d}$. By (ii), $\left(\mu^{*}\right)_{d}=\mu_{d}$. Consequently, $v=v_{c}+v_{d} \leq\left(\mu^{*}\right)_{c}+\mu_{d}=\left(\mu^{*}\right)_{c}+\left(\mu^{*}\right)_{d}=\mu^{*}$. To prove (v), let us observe that $-\mu^{-} \in \mathcal{G}$, because $-R \mu^{-}$is a solution of (4.1) with $\mu$ replaced by $-\mu^{-}$. Hence, by (iv), $-\mu^{-} \leq \mu^{*}$, from which we easily get (v). To show (vi), let us observe that $\mu^{*} \in \mathcal{G}$, and by (i), $\mu^{*} \leq v$. Hence $\mu^{*} \leq v^{*}$ by (iv).

Proposition 5.5 A measure $\mu \in \mathbb{M}$ is good if and only if the sequence $\left\{f_{n}\left(X, u_{n}(X)\right)\right\}$ considered in the proof of Theorem 5.2 is uniformly integrable under the measure $d t \otimes P_{x}$ for $m$-a.e. $x \in E$.

Proof From the proof of Theorem 5.2 we know that $f_{n}\left(X, u_{n}(X)\right) \rightarrow f\left(X, u^{*}(X)\right), d t \otimes P_{x^{-}}$ a.e. for $m$-a.e. $x \in E$ and

$$
u_{n}(x)=R \mu_{c}+E_{x} \int_{0}^{\zeta} f_{n}\left(X_{r}, u_{n}\left(X_{r}\right)\right) d r+E_{x} \int_{0}^{\zeta} d A_{r}^{\mu_{d}}
$$

for q.e. $x \in E$. If $\left\{f_{n}\left(X, u_{n}(X)\right)\right\}$ is uniformly integrable then letting $n \rightarrow \infty$ in (5.4) shows that for q.e. $x \in E$,

$$
u^{*}(x)=R \mu_{c}+E_{x} \int_{0}^{\zeta} f\left(X_{r}, u^{*}\left(X_{r}\right)\right) d r+E_{x} \int_{0}^{\zeta} d A_{r}^{\mu_{d}},
$$

i.e. $\mu$ is a good measure. If $\mu \in \mathcal{G}$ then there exists a solution $u$ of (4.1), i.e.

$$
u(x)=R \mu_{c}+E_{x} \int_{0}^{\zeta} f\left(X_{r}, u\left(X_{r}\right)\right) d r+E_{x} \int_{0}^{\zeta} d A_{r}^{\mu_{d}}
$$

for q.e. $x \in E$. Of course, $u$ is a subsolution of (4.1), so by Theorem 5.2, $u=u^{*}$ and $u_{n} \searrow u$. By this and (5.4),

$$
E_{x} \int_{0}^{\zeta} f_{n}\left(X_{r}, u_{n}\left(X_{r}\right)\right) d r \rightarrow E_{x} \int_{0}^{\zeta} f\left(X_{r}, u\left(X_{r}\right)\right) d r
$$

for q.e. $x \in E$. Since $f_{n}\left(X, u_{n}(X)\right) \rightarrow f(X, u(X)), d t \otimes d P_{x}$-a.e. for q.e. $x \in E$ and $f_{n}\left(X, u_{n}(X)\right) \leq 0$, applying Vitali's theorem shows that the sequence $\left\{f_{n}\left(X, u_{n}(X)\right)\right\}$ is uniformly integrable under the measure $d t \otimes P_{x}$ for q.e. $x \in E$, and hence for $m$-a.e. $x \in E$.

Proposition 5.6 If $v \in \mathbb{M}, \mu \in \mathcal{G}$ and $v \leq \mu$, then $v \in \mathcal{G}$.

Proof Let $\left\{u_{n}\right\}$ be the sequence of functions of Theorem 5.2 associated with $\mu$ and let $\left\{v_{n}\right\}$ be a sequence constructed as $\left\{u_{n}\right\}$ but for $\mu$ replaced by $v$. By Proposition 4.2, $v_{n} \leq u_{n}$ q.e. Consequently, $f_{n}\left(\cdot, u_{n}\right) \leq f\left(\cdot, v_{n}\right) \leq 0$ q.e. Since $\mu \in \mathcal{G}$, we know from Proposition 5.5 that the sequence $\left\{f_{n}\left(X, u_{n}(X)\right)\right\}$ is uniformly integrable under the measure $d t \otimes P_{x}$ for $m$-a.e. $x \in E$. Therefore $\left\{f_{n}\left(X, v_{n}(X)\right)\right\}$ has the same property. By Proposition 5.5, this implies that $v \in \mathcal{G}$.

Corollary 5.7 If $\mu \in \mathbb{M}$ and $\mu^{+} \in \mathcal{G}$, then $\mu \in \mathcal{G}$.

Proof Follows immediately from Proposition 5.6 and the fact that $\mu \leq \mu^{+}$.

Corollary 5.8 If $\mu_{1}, \mu_{2} \in \mathcal{G}$, then $\mu_{1} \vee \mu_{2} \in \mathcal{G}$.

Proof Let $\mu=\mu_{1} \vee \mu_{2}$. Since $\mu_{1} \leq \mu, \mu_{2} \leq \mu$ and $\mu_{1}, \mu_{2} \in \mathcal{G}$, it follows from Proposition 5.4(iv) that $\mu_{1} \leq \mu^{*}$ and $\mu_{2} \leq \mu^{*}$. Hence $\mu \leq \mu^{*}$. On the other hand, by Proposition 5.4(i), $\mu^{*} \leq \mu$, so $\mu=\mu^{*}$, i.e. $\mu \in \mathcal{G}$. 
Corollary 5.9 The set $\mathcal{G}$ is convex.

Proof Let $\mu_{1}, \mu_{2} \in \mathcal{G}$. Then $\mu_{1} \vee \mu_{2} \in \mathcal{G}$ by Corollary 5.8. But for every $t \in[0,1]$, $t \mu_{1}+(1-t) \mu_{2} \leq \mu_{1} \vee \mu_{2}$, so by Proposition 5.6, $t \mu_{1}+(1-t) \mu_{2} \in \mathcal{G}, t \in[0,1]$.

Set $\mathcal{G}_{\rho}=\mathcal{G} \cap \mathcal{M}_{\rho}$.

Theorem 5.10 We have

(i) $\left\|\mu-\mu^{*}\right\|_{\rho}=\min _{v \in \mathcal{G}_{\rho}}\|\mu-v\|_{\rho}$ for every $\mu \in \mathcal{M}_{\rho}$,

(ii) if $\mu_{1}, \mu_{2} \in \mathbb{M}$ and $\mu_{1} \perp \mu_{2}$, then $\left(\mu_{1}+\mu_{2}\right)^{*}=\mu_{1}^{*}+\mu_{2}^{*}$,

(iii) $(\mu \wedge v)^{*}=\mu^{*} \wedge v^{*}$ and $(\mu \vee v)^{*}=\mu^{*} \vee v^{*}$ for every $\mu, v \in \mathbb{M}$,

(iv) $\left(\mu^{*}-v^{*}\right)^{+} \leq(\mu-v)^{+}$for every $\mu, v \in \mathbb{M}$.

Proof It suffices to repeat step by step the reasoning from the proofs of Corollary 6 and Theorems $8-10$ in [5].

Theorem 5.11 Let $\mu \in \mathbb{M}$. The following conditions are equivalent:

(i) $\mu \in \mathcal{G}$,

(ii) $\mu^{+} \in \mathcal{G}$,

(iii) $\mu_{c} \in \mathcal{G}$,

(iv) $\mu=g-A v$ for some functions $g$, $v$ on $E$ such that $g \cdot m \in \mathbb{M}$ and $f(\cdot, v) \cdot m \in \mathbb{M}$.

Proof That (i) is equivalent to (ii) follows from Corollaries 5.7 and 5.8. That (ii) implies (iii) follows from the fact that $\mu_{c} \leq \mu^{+}$and Proposition 5.6. Suppose that $\mu_{c} \in \mathcal{G}$. Since $\mu_{d} \in \mathcal{G}$ and $\mu^{+}=\mu_{d} \vee \mu_{c}$, it follows from Corollary 5.8 that $\mu^{+} \in \mathcal{G}$. Thus (iii) implies (ii). Of course (i) implies (iv). Suppose now that (i) is satisfied. Then

$$
-A v=f(\cdot, v)+(\mu-g-f(\cdot, v)) .
$$

Hence $\mu-g-f(\cdot, v) \in \mathcal{G}$, and consequently $(\mu-g-f(\cdot, v))_{c}=\mu_{c} \in \mathcal{G}$, because we already know that (i) implies (iii). Hence $\mu \in \mathcal{G}$, because we also know that (iii) implies (i).

Set

$$
L(E ; m)=\{f \in \mathcal{B}(E): f \cdot m \in \mathbb{M}\}
$$

$$
\mathcal{A}(f)=\{\mu \in \mathbb{M}: f(\cdot, R \mu) \in L(E ; m)\}, \quad \mathcal{A}_{\rho}=\left\{\mu \in \mathcal{M}_{\rho} ; f(\cdot, R \mu) \in L^{1}(E ; \rho \cdot m)\right\} .
$$

Corollary 5.12 We have

(i) $\mathcal{G}+\mathbb{M} \cap S \subset \mathcal{G}$,

(ii) $\mathcal{A}(f)+L(E ; m)=\mathcal{G}$,

(iii) $\mathcal{A}_{\rho}(f)+L^{1}(E ; \rho \cdot m)=\mathcal{G}_{\rho}$.

Let us consider the following hypothesis:

(A) for every $\theta \in[0,1), c \geq 0$ there exist $\alpha(c, \theta), \beta(c, \theta) \geq 0$ such that

$$
|f(x, \theta u+c)| \leq \alpha(c, \theta)|f(x, u)|+\beta(c, \theta), \quad x \in E, u \in \mathbb{R} .
$$

Theorem 5.13 Let $\rho \in L^{1}(E ; m)$. If $(A)$ is satisfied then $\overline{\mathcal{A}_{\rho}(f)}=\mathcal{G}_{\rho}$, where the closure is taken in the space $\left(\mathcal{M}_{\rho},\|\cdot\|_{\rho}\right)$. 
Proof First we show that $\mathcal{G}_{\rho}$ is a closed subset of $\left(\mathcal{M}_{\rho},\|\cdot\|_{\rho}\right)$. Let $\left\{\mu_{n}\right\} \subset \mathcal{G}_{\rho}$ be a sequence such that $\mu_{n} \rightarrow \mu$ in $\left(\mathcal{M}_{\rho},\|\cdot\|_{\rho}\right)$ for some $\mu \in \mathcal{M}_{\rho}$. Let $u_{n}$ denote a solution of (4.1) with $\mu$ replaced by $\mu_{n}$ and let $\psi$ be a strictly positive Borel function on $E$ such that $R \psi \leq \rho, m$-a.e. Let us observe that

$$
\left(R\left|\mu_{n}-\mu_{m}\right|, \psi\right) \leq\left\|\mu_{n}-\mu_{m}\right\|_{\rho}, \quad n, m \geq 1
$$

By Proposition 4.4,

$$
\left(R\left|f_{u_{n}}-f_{u_{m}}\right|, \psi\right) \leq\left\|\mu_{n}-\mu_{m}\right\|_{\rho}, \quad n, m \geq 1 .
$$

Adding (5.5) to (5.6) gives

$$
\int_{E}\left|u_{n}(x)-u_{m}(x)\right| \psi(x) m(d x) \leq 2\left\|\mu_{n}-\mu_{m}\right\|_{\rho}, \quad n, m \geq 1 .
$$

Therefore there exists $u \in L^{1}(E ; \psi \cdot m)$ such that $u_{n} \rightarrow u$ in $L^{1}(E ; \psi \cdot m)$. By the definition of a solution of (4.1),

$$
u_{n}=R f_{u_{n}}+R \mu_{n}, \quad m \text {-a.e. }
$$

By (5.5) and (5.6) the right-hand side of the above equation converges in $L^{1}(E ; \psi \cdot m)$ to $R f_{u}+R \mu$. Hence

$$
u=R f_{u}+R \mu, \quad m \text {-a.e., }
$$

which implies that $\mu \in \mathcal{G}_{\rho}$, and hence that $\mathcal{G}_{\rho}$ is closed. Therefore $\overline{\mathcal{A}_{\rho}(f)} \subset \mathcal{G}_{\rho}$, because $\mathcal{A}_{\rho}(f) \subset \mathcal{G}_{\rho}$ by Theorem 5.11. Now suppose that $\mu \in \mathcal{G}_{\rho}$. Then there exists a solution $u$ of (4.1). Let $\theta_{n}=\left(1-\frac{1}{n}\right)$ and let $\left\{F_{n}\right\}$ be a nest such that $c(n):=\left\|R\left(\mathbf{1}_{F_{n}} f(\cdot, u)\right)\right\|_{\infty}<\infty$ (such a nest exists, because $f(\cdot, u) \in \mathcal{M}_{\rho} \subset \mathbb{M}$ ). Let $\mu_{n}=-\theta_{n} A u-\mathbf{1}_{F_{n}} f(\cdot, u)$. By (A),

$$
\left|f\left(x, R \mu_{n}(x)\right)\right| \leq \alpha\left(c(n), \theta_{n}\right)|f(x, u(x))|+\beta\left(c(n), \theta_{n}\right), \quad x \in E .
$$

By Proposition 4.8, $f_{u} \in L^{1}(E ; \rho \cdot m)$. Therefore from (5.7) it follows that $\mu_{n} \in \mathcal{A}_{\rho}(f)$. Since it is clear that $\left\|\mu_{n}-\mu\right\|_{\rho} \rightarrow 0$, we have $\mu \in \overline{\mathcal{A}_{\rho}(f)}$, which completes the proof.

\section{Inverse maximum principle and Kato's inequality}

In this section we consider the linear equation (3.1). The following theorem generalizes the inverse maximum principle proved by H. Brezis and A.C. Ponce in [6] in case $A$ is the Laplace operator on a bounded domain in $\mathbb{R}^{d}$.

Theorem 6.1 Let $\mu \in \mathbb{M}$ and $u$ be a solution of (3.1). If $u \geq 0$ then $\mu_{c} \geq 0$.

Proof Assume that $u \geq 0$. Let $\left\{\tau_{k}\right\}$ be a reducing sequence for $u$. By the definition of a solution of (3.1), for every $\alpha \geq 0$,

$$
\lim _{k \rightarrow \infty} E_{x} e^{-\alpha \tau_{k}} u\left(X_{\tau_{k}}\right)=R_{\alpha} \mu_{c}(x)
$$

for q.e. $x \in E$. In particular, $R_{\alpha} \mu_{c}(x) \geq 0$ for q.e. $x \in E$, and hence, by [3, PropositionII.3.2], $R_{\alpha} \mu_{c} \geq 0$ everywhere. That $\mu_{c} \geq 0$ now follows from Lemma 3.5.

Proposition 6.2 Assume that $\mu \in \mathbb{M}$. Let $u$ be a solution of (3.1) and let $\varphi$ be a positive convex Lipschitz continuous function on $\mathbb{R}$ such that $\varphi(0)=0$. Then $A \varphi(u) \in \mathbb{M}$. Moreover,

$$
\|A \varphi(u)\|_{\rho} \leq \operatorname{Lip}(\varphi)\|\mu\|_{\rho} .
$$


Proof Let $\left\{\tau_{k}\right\}$ be a reducing sequence for $u$. By the definition of a probabilistic solution of (3.1),

$$
u\left(X_{t}\right)=u\left(X_{0}\right)-\int_{0}^{t} d A_{r}^{\mu_{d}}+\int_{0}^{t} d M_{r}, \quad t \geq 0
$$

for some local MAF $M$. By the Itô-Meyer formula,

$$
\begin{aligned}
\varphi(u)\left(X_{t}\right)= & \varphi(u)\left(X_{0}\right)-\int_{0}^{t} \varphi^{\prime}\left(u\left(X_{r}\right)\right) d A_{r}^{\mu_{d}} \\
& +\int_{0}^{t} d A_{r}+\int_{0}^{t} \varphi^{\prime}\left(u\left(X_{r-}\right)\right) d M_{r}, \quad t \geq 0
\end{aligned}
$$

for some increasing process $A$, where $\varphi^{\prime}$ is the left derivative of $\varphi$. Let $A^{p}$ denote the dual predictable projection of $A$ (one can find a version of $A^{p}$ which is independent of $x$; see [9]). Since $A^{p}$ is predictable, it is continuous, because the filtration $\left(\mathcal{F}_{t}\right)$ is quasi-left continuous. Therefore there exists a positive smooth measure $v$ such that $A^{p}=A^{v}$. For q.e. $x \in E$ we have

$$
\begin{aligned}
E_{x} \int_{0}^{\zeta} d A_{r}^{v} & =\lim _{k \rightarrow \infty} E_{x} \int_{0}^{\tau_{k}} d A_{r}^{v} \leq \lim _{k \rightarrow \infty}\left(E_{x} \varphi\left(u\left(X_{\tau_{k}}\right)\right)+E_{x} \int_{0}^{\tau_{k}} \varphi^{\prime}\left(u\left(X_{r}\right)\right) d A_{r}^{\mu_{d}}\right) \\
& \leq \operatorname{Lip}(\varphi) \lim _{k \rightarrow \infty}\left(E_{x}\left|u\left(X_{\tau_{k}}\right)\right|+E_{x} \int_{0}^{\tau_{k}} d A_{r}^{\left|\mu_{d}\right|}\right) \leq 2 \operatorname{Lip}(\varphi) R|\mu|(x) .
\end{aligned}
$$

Thus $v \in \mathbb{M}$. Write

$$
v_{1}(x)=R v(x), \quad v_{2}(x)=R \mu_{d}^{-}(x), \quad x \in E
$$

and observe that

$$
\varphi(u)\left(X_{t}\right)+v_{1}\left(X_{t}\right)+v_{2}\left(X_{t}\right)=\varphi(u)(x)+v_{1}(x)+v_{2}(x)-\int_{0}^{t} d A^{\mu_{d}^{+}}+\int_{0}^{t} d \bar{M}_{r}, \quad t \geq 0
$$

for some local MAF $\bar{M}$. Set $w=\varphi(u)+v_{1}+v_{2}$. From the above equation and the fact that $w \geq 0$ it follows that $w(X)$ is a supermartingale. Therefore $w$ is an excessive function. On the other hand,

$$
w \leq|\varphi(u)|+v_{1}+v_{2} \leq \operatorname{Lip}(\varphi)|u|+R v+R \mu_{d}^{-} \leq \operatorname{Lip}(\varphi) R|\mu|+R v+R \mu_{d} .
$$

Therefore by [14, Proposition 3.9] there exists a positive $\beta \in \mathbb{M}$ such that $w=R \beta$. This implies that $A \varphi(u)=\beta-v-\mu_{d}^{-} \in \mathbb{M}$. By (6.1) and the assumptions on $\varphi$,

$$
\begin{aligned}
\varphi(u)(x) & =E_{x} \varphi(u)\left(X_{\tau_{k}}\right)+E_{x} \int_{0}^{\tau_{k}} \varphi^{\prime}\left(u\left(X_{r}\right)\right) d A_{r}^{\mu_{d}}-E_{x} \int_{0}^{\tau_{k}} d A_{r}^{v} \\
& \leq \operatorname{Lip}(\varphi)\left(E_{x}|u|\left(X_{\tau_{k}}\right)+E_{x} \int_{0}^{\tau_{k}} d A_{r}^{\left|\mu_{d}\right|}\right)
\end{aligned}
$$

for q.e. $x \in E$. Letting $k \rightarrow \infty$ and applying Lemma 4.6 we get the desired result.

The following version of Kato's inequality was proved by H. Brezis and A.C. Ponce [6] (see also H. Brezis, M. Marcus and A.C. Ponce [5]) in case $A$ is the Laplace operator on a bounded domain in $\mathbb{R}^{d}$ ). 
Theorem 6.3 Let $u$ be a solution of (3.1). Then $A u^{+} \in \mathbb{M}$ and

$$
\begin{gathered}
\mathbf{1}_{\{u>0\}}(A u)_{d} \leq\left(A u^{+}\right)_{d}, \\
(A u)_{c}^{+}=\left(A u^{+}\right)_{c} .
\end{gathered}
$$

Proof By Proposition 6.2 and (6.1), $A u^{+} \in \mathbb{M}$ and there exist positive $v, l \in \mathbb{M}$ such that $v \perp$ Cap, $l \ll$ Cap and

$$
-A u^{+}=v+\mathbf{1}_{\{u>0\}} \mu_{d}-l .
$$

By the resolvent identity, for every $\alpha \geq 0$ we have

$$
u=R_{\alpha}(\mu+\alpha u), \quad u^{+}=R_{\alpha}\left(v+\mathbf{1}_{\{u>0\}} \mu_{d}-l+\alpha u^{+}\right) .
$$

It is clear that

$$
R_{\alpha}\left(v+\mathbf{1}_{\{u>0\}} \mu_{d}-l+\alpha u^{+}\right) \leq R_{\alpha}(\mu+\alpha u)^{+} .
$$

Hence

$$
R_{\alpha}\left(v+\mathbf{1}_{\{u>0\}} \mu_{d}-l\right) \leq R_{\alpha}\left[(\mu+\alpha u)^{+}-\alpha u^{+}\right] \leq R_{\alpha} \mu^{+} .
$$

By Lemma 3.5,

$$
v+\mathbf{1}_{\{u>0\}} \mu_{d}-l \leq \mu^{+} .
$$

Taking the diffuse part of the above inequality we get (6.2). Taking the concentrated part we get

$$
v \leq \mu_{c}^{+} .
$$

On the other hand, since $u^{+}-u \geq 0$, it follows from Theorem 6.1 that

$$
\left(v+\mathbf{1}_{\{u>0\}} \mu_{d}-l-\mu\right)_{c} \geq 0,
$$

which implies that $v \geq \mu_{c}^{+}$. When combined with (6.4) this gives (6.2).

Remark 6.4 Applying in the proof of Theorem 6.1 the Itô-Meyer formula with right derivative of the function $u \mapsto u^{+}$we obtain (6.5) with $\mathbf{1}_{\{u>0\}}$ replaced by $\mathbf{1}_{\{u \geq 0\}}$. As a result, we get (6.2) with $\mathbf{1}_{\{u>0\}}$ replaced by $\mathbf{1}_{\{u \geq 0\}}$.

\section{Equations with polynomial nonlinearity}

In this section we give a necessary and sufficient condition on $\mu$ ensuring the existence of a solution of (4.1) with $f$ satisfying the condition

$$
|f(x, u)| \leq c u^{p}, \quad x \in E, u \geq 0
$$

for some constants $c \geq 0, p>1$. We also calculate the reduced measure in the case where $f(x, u)=-u^{p}$. In our study a primary role will be played by a new capacity $\mathrm{Cap}_{A, p}$, which we define below.

Let $p \geq 1$. By the Riesz-Thorin interpolation theorem one can extend the semigroup $\left\{T_{t}, t \geq 0\right\}$ from $L^{2}(E ; m) \cap L^{p}(E ; m)$ to $L^{p}(E ; m)$. We denote the extended semigroup by $\left\{T_{t}^{\bar{p}}, t \geq 0\right\}$, whereas by $\left\{R_{\alpha}^{p}, \alpha>0\right\}$ we denote its resolvent. Let $\left(A_{p}, D\left(A_{p}\right)\right)$ be the operator generated by $\left\{T^{p}\right\}$. It is well known that $D\left(A_{p}\right)=R_{1}^{p}\left(L^{p}(E ; m)\right)$. We set 
$D_{+}\left(A_{p}\right)=R_{1}^{p}\left(L^{p,+}(E ; m)\right)$. Each element of $D_{+}\left(A_{p}\right)$ is defined pointwise via the resolvent kernel. Let $V_{p}$ denote the space $D\left(A_{p}\right)$ equipped with the norm

$$
\|u\|_{V_{p}}=\left\|A_{p} u\right\|_{L^{p}(E ; m)}+\|u\|_{L^{p}(E ; m)} .
$$

We define the capacity of $B \subset E$ as

$$
\operatorname{Cap}_{A, p}(B)=\inf \left\{\|\eta\|_{V_{p}}^{p}: \eta \in D_{+}\left(A_{p}\right), \eta \geq \mathbf{1}_{B}\right\} .
$$

It is an elementary check that $\operatorname{Cap}_{A, p}$ is subadditive and increasing (see, e.g., [1, Proposition 2.3.6]). We say that $\mu \in V_{p}^{\prime} \cap \mathbb{M}^{+}$if for every $\eta \in V_{p}^{+}$,

$$
(\eta, \mu) \leq c\|\eta\|_{V_{p}} .
$$

In the rest of the section we assume that $p>1$. By $p^{\prime}$ we denote the Hölder conjugate to p.

Proposition 7.1 If $\mu \in V_{p}^{\prime} \cap \mathbb{M}^{+}$then $\mu$ is a good measure relative to the function $f(u)=$ $-|u|^{p^{\prime}}$.

Proof Let $u$ be a solution of the equation

$$
(I-A) u=\mu .
$$

Then $u \in L^{p^{\prime}}(E ; m) \cap L(E ; m)$. Indeed, the fact that $u \in L(E ; m)$ follows from the inequality $R u \leq R \mu$. Now, for $f \in L^{p,+}(E ; m)$ set $\eta=R_{1}^{p} f$. Then

$$
\begin{aligned}
\int_{E} u f d m & =\int_{E} u\left(I-A_{p}\right) \eta d m=\int_{E}\left(I-A_{p}\right) u \eta d m=\int_{E} \eta d \mu \\
& \leq c\|\eta\|_{V_{p}}=c\left(\left\|A_{p} \eta\right\|_{L^{p}(E ; m)}+\|\eta\|_{L^{p}(E ; m)}\right) \leq 2 c\|f\|_{L^{p}(E ; m)},
\end{aligned}
$$

which shows that $u \in L^{p^{\prime}}(E ; m)$. That $\mu$ is a good measure relative to $f(u)=-|u|^{p^{\prime}}$ now follows from Theorem 5.11.

Lemma 7.2 Let $u \in D_{+}\left(A_{p}\right)$. Then for every $\lambda>0$,

$$
\operatorname{Cap}_{A, p}(u \geq \lambda) \leq \lambda^{-p}\|u\|_{V_{p}}^{p} .
$$

Proof Let $B=\{u \geq \lambda\}$. Then $\lambda^{-1} u \geq \mathbf{1}_{B}$, so the required inequality follows immediately from the definition of $\operatorname{Cap}_{A, p}$.

Lemma 7.3 Let $\mu \in \mathcal{M}_{b}^{+}$. If $\mu \leq c \cdot$ Cap $_{A, p}$ for some $c \geq 0$, then $\mu \in V_{p}^{\prime}$.

Proof Let $\eta \in V_{p}^{+}$. By our assumptions on $\mu$ and Lemma 7.2, for any $\eta \in V_{p}^{+}$with $\|\eta\|_{V_{p}}=1$ we have

$$
\begin{aligned}
\int_{E} \eta d \mu \leq \mu(E)+\sum_{k=0}^{\infty} 2^{k+1} \mu\left(\eta \geq 2^{k}\right) & \leq \mu(E)+c \sum_{k=0}^{\infty} 2^{k+1} \operatorname{Cap}_{A, p}\left(\eta \geq 2^{k}\right) \\
& \leq \mu(E)+c \sum_{k=0}^{\infty} 2^{k(1-p)+1}<\infty
\end{aligned}
$$

which proves the lemma. 
Lemma 7.4 Let $\mu \in \mathcal{M}_{b}^{+}$and $\mu \ll \operatorname{Cap}_{A, p}$. Then there exists a decreasing sequence $\left\{G_{n}\right\}$ of Borel subsets of $E$ such that

$$
\lim _{n \rightarrow \infty} \operatorname{Cap}_{A, p}\left(G_{n}\right)=0, \quad \lim _{n \rightarrow \infty} \mu\left(G_{n}\right)=0, \quad \mathbf{1}_{E \backslash G_{n}} \cdot \mu \leq 2^{n} \operatorname{Cap}_{A, p}, \quad n \geq 1 .
$$

Proof It is enough to repeat step by step the proof of [12, Lemma 2.2.9], the only difference being in the fact that we choose the sets $B_{n}$ appearing in the proof of [12, Lemma 2.2.9] as Borel sets.

As a corollary to Lemma 7.4 we get the following proposition.

Proposition 7.5 A measure $\mu \in \mathbb{M}^{+}$satisfies $\mu \ll$ Cap ${ }_{A, p}$ if and only if there exists an increasing sequence $\left\{E_{n}\right\}$ of Borel subsets of $E$ such that $\mathbf{1}_{E_{n}} \cdot \mu \in V_{p}^{\prime} \cap \mathbb{M}^{+}$for $n \in \mathbb{N}$ and $\mu\left(E \backslash \bigcup_{n \geq 1} E_{n}\right)=0$.

Theorem 7.6 Assume (7.1). If $\mu \in \mathbb{M}$ and $\mu^{+} \ll$ Cap $_{A, p^{\prime}}$ then $\mu \in \mathcal{G}$.

Proof By Theorem 5.11 we may assume that $\mu \geq 0$. By Lemma 4.10 there exists a strictly positive bounded excessive function $\rho$ such that $\mu \in \mathcal{M}_{\rho}^{+}$, and by Proposition 7.5 there exists a sequence $\left\{\mu_{n}\right\} \subset V_{p^{\prime}}^{\prime} \cap \mathbb{M}^{+}$such that $\lim _{n \rightarrow \infty}\left\|\mu_{n}-\mu\right\|_{\rho}=0$. Therefore it is enough to show that $\mu_{n} \in \mathcal{G}$. But this follows from Proposition 7.1.

Corollary 7.7 Assume that $\mu \in \mathbb{M}$ and an let $f(x, u)=-u^{p}, x \in E, u \geq 0$. Then $\mu \in \mathcal{G}$ if and only if $\mu^{+} \ll \operatorname{Cap}_{A, p^{\prime}}$.

Proof Sufficiency follows from Theorem 7.6. Suppose that $\mu \in \mathcal{G}$. By Theorem 5.11, $\mu^{+} \in$ $\mathcal{G}$. By Proposition 5.6 and closedness of $\mathcal{G}$ we may assume that $\mu^{+}$is bounded. Assume that $\mathrm{Cap}_{A, p^{\prime}}(B)=0$ for some Borel set $B \subset E$. Then there exists a sequence $\left\{\eta_{n}\right\} \subset V_{p^{\prime}}^{+}$such that $\left\|\eta_{n}\right\|_{V_{p^{\prime}}} \rightarrow 0, \sup _{n \geq 1} \eta_{n} \leq c$ for some $c>0$ and $\eta_{n} \geq \mathbf{1}_{B}$. Let $u$ be a solution of (4.1) with $\mu$ replaced by $\mu^{+}$. Then $u \in L^{p}(E ; m)$ by Proposition 4.8. Therefore

$$
\begin{aligned}
\mu^{+}(B) & \leq\left(\eta_{n}, \mu^{+}\right)=\left(u^{p}, \eta_{n}\right)+\left(u,-A_{p} \eta_{n}\right) \\
& \leq\left(u^{p}, \eta_{n}\right)+\|u\|_{L^{p}(E ; m)}\left\|A_{p} \eta_{n}\right\|_{L^{p^{\prime}}(E ; m)} \leq\left(u^{p}, \eta_{n}\right)+\|u\|_{L^{p}(E ; m)}\left\|\eta_{n}\right\|_{V_{p^{\prime}}}
\end{aligned}
$$

for every $n \in \mathbb{N}$, which forces $\mu^{+}(B)=0$.

Corollary 7.8 Let the assumptions of Corollary 7.7 hold. Let $\mu_{C a p_{A, p^{\prime}}}^{+}$denote the absolutely continuous part, with respect to Cap $_{A, p^{\prime}}$, of the measure $\mu^{+}$. Then

$$
\mu^{*}=\mu_{C a p_{A, p^{\prime}}}^{+}-\mu^{-} .
$$

Proof It suffices to repeat step by step the proof of [5, Theorem 16].

Remark 7.9 Let us note that from [1, Proposition 2.3.13] (see also [15]) it follows that for all $p>1, \alpha \in(0,1]$ and open bounded set $D \subset \mathbb{R}^{d}$,

$$
c_{1} \operatorname{Cap}_{\alpha, p}^{D}(B) \leq \operatorname{Cap}_{A, p}(B) \leq c_{2} \operatorname{Cap}_{\alpha, p}^{D}(B), \quad B \subset D,
$$

where $A=\Delta^{\alpha}$ on $D$ with zero boundary condition (see Remark 4.13) and for a compact $K \subset D$ the capacity $\operatorname{Cap}_{\alpha, p}^{D}(K)$ is defined by (1.5). 
Open Access This article is distributed under the terms of the Creative Commons Attribution 4.0 International License (http://creativecommons.org/licenses/by/4.0/), which permits unrestricted use, distribution, and reproduction in any medium, provided you give appropriate credit to the original author(s) and the source, provide a link to the Creative Commons license, and indicate if changes were made.

\section{References}

1. Adams, D.R., Hedberg, L.I.: Function spaces and potential theory. Springer-Verlag, Berlin (1996)

2. Bénilan, P., Brezis, H.: Nonlinear problems related to the Thomas-Fermi equation. J. Evol. Equ. 3, 673770 (2004)

3. Blumenthal, M.R., Getoor, R.K.: Markov processes and potential theory. Dover Publications, New York (2007)

4. Brezis, H., Marcus, M., Ponce, A.C.: A new concept of reduced measure for nonlinear elliptic equations. C. R. Math. Acad. Sci. 339, 169-174 (2004)

5. Brezis, H., Marcus, M., Ponce, A.C.: Nonlinear elliptic equations with measures revisited. In: Mathematical Aspects of Nonlinear Dispersive Equations (J. Bourgain, C. Kenig, S. Klainerman, eds.), Annals of Mathematics Studies, 163, Princeton University Press, Princeton, NJ, 55-110 (2007)

6. Brezis, H., Ponce, A.C.: Kato's inequality when $\Delta u$ is a measure. C. R. Math. Acad. Sci. Paris 338, 599-604 (2004)

7. Brezis, H., Strauss, W.A.: Semilinear second-order elliptic equations in $L^{1}$. J. Math. Soc. Japan 25, 565-590 (1973)

8. Chen, H., Véron, L.: Semilinear fractional elliptic equations involving measures. J. Differ. Equ. 257, 1457-1486 (2014)

9. Çinlar, E., Jacod, J., Protter, P., Sharpe, M.J.: Semimartingales and markov processes. Z. Wahrscheinlichkeitstheorie verw. Gebiete 54, 161-219 (1980)

10. Dellacherie, C., Meyer, P.A.: Probabilities and potential B. North-Holland, Amsterdam (1982)

11. Dellacherie, C., Meyer, P.A.: Probabilities and Potential C. North-Holland, Amsterdam (1988)

12. Fukushima, M., Oshima, Y., Takeda, M.: Dirichlet forms and symmetric Markov processes. Walter de Gruyter, Berlin (1994)

13. Fukushima, M., Sato, K., Taniguchi, S.: On the closable parts of pre-Dirichlet forms and the fine supports of underlying measures. Osaka J. Math. 28, 517-535 (1991)

14. Getoor, R.K., Glover, J.: Riesz decomposition in Markov process theory. Trans. Amer. Math. Soc. 285, 107-132 (1984)

15. Gurarie, D.: On $L^{p}$-domains of fractional powers of singular elliptic operators and Kato's conjecture. J. Oper. Theory 27, 193-203 (1992)

16. Jakubowski, A., Mémin, J., Pages, G.: Convergence en loi des suites d'intégrales stochastiques sur l'espace $D^{1}$. Probab. Theory Relat. Fields 81, 111-137 (1989)

17. Klimsiak, T., Rozkosz, A.: Dirichlet forms and semilinear elliptic equations with measure data. J. Funct. Anal. 265, 890-925 (2013)

18. Klimsiak, T., Rozkosz, A.: Renormalized solutions of semilinear equations involving measure data and operator corresponding to Dirichlet form. NoDEA Nonlinear Differ. Equ. Appl. 22, 1911-1934 (2015)

19. Klimsiak, T., Rozkosz, A.: Semilinear elliptic equations with measure data and quasi-regular Dirichlet forms. Colloq. Math. 145, 35-67 (2016). doi:10.4064/cm6466-10-2015

20. Klimsiak, T., Rozkosz, A.: On the structure of bounded smooth measures associated with quasi-regular Dirichlet form. Available at arXiv:1410.4927

21. Konishi, Y.: Une remarque sur la perturbation d'opérateurs $m$-accrétifs dans un espace de Banach. Proc. Japan Acad. 48, 157-160 (1972)

22. Kulczycki, T.: Properties of Green function of symmetric stable processes. Probab. Math. Statist. 17, 339-364 (1997)

23. Ma, Z., Röckner, M.: Introduction to the theory of (non-symmetric) Dirichlet forms. Springer-Verlag, Berlin (1992)

24. Oshima, Y.: Semi-Dirichlet forms and Markov processes. Walter de Gruyter, Berlin (2013)

25. Protter, P.: Stochastic integration and differential equations, 2nd edn. Springer, Berlin (2004)

26. Sharpe, M.: General theory of Markov processes. Academic Press, New York (1988)

27. Stampacchia, G.: Le problème de Dirichlet pour les équations elliptiques du second ordre à coefficients discontinus. Ann. Inst. Fourier (Grenoble) 15, 189-258 (1965)

28. Véron, L.: Elliptic equations involving measures. Stationary partial differential equations, Vol. I, 593-712. Handb. Differ. Equ., North- Holland, Amsterdam (2004) 